\title{
An Analysis of an Ostensible Anticyclonic Tornado from 9 May 2016 Using High-Resolution, Rapid-Scan Radar Data
}

\author{
JEFFREY C. SNYDER \\ NOAA/OAR National Severe Storms Laboratory, Norman, Oklahoma \\ HOWARD B. BLUESTEIN AND ZACHARY B. WIENHOFF ${ }^{a}$ \\ School of Meteorology, University of Oklahoma, Norman, Oklahoma
}

CHARLES M. KUSTER

NOAA/OAR National Severe Storms Laboratory, and Cooperative Institute for Mesoscale Meteorological Studies, University of Oklahoma, Norman, Oklahoma

DYLAN W. REIF

School of Meteorology, University of Oklahoma, Norman, Oklahoma

(Manuscript received 7 April 2020, in final form 15 June 2020)

\begin{abstract}
Tornadic supercells moved across parts of Oklahoma on the afternoon and evening of 9 May 2016. One such supercell, while producing a long-lived tornado, was observed by nearby WSR-88D radars to contain a strong anticyclonic velocity couplet on the lowest elevation angle. This couplet was located in a very atypical position relative to the ongoing cyclonic tornado and to the supercell's updraft. A storm survey team identified damage near where this couplet occurred, and, in the absence of evidence refuting otherwise, the damage was thought to have been produced by an anticyclonic tornado. However, such a tornado was not seen in near-ground, high-resolution radar data from a much closer, rapid-scan, mobile radar. Rather, an elongated velocity couplet was observed only at higher elevation angles at altitudes similar to those at which the WSR-88D radars observed the strong couplet. This paper examines observations from two WSR-88D radars and a mobile radar from which it is argued that the anticyclonic couplet (and a similar one $\sim 10 \mathrm{~min}$ later) were actually quasi-horizontal vortices centered $\sim 1-1.5 \mathrm{~km}$ AGL. The benefits of having data from a radar much closer to the convective storm being sampled (e.g., better spatial resolution and near-ground data coverage) and providing more rapid volume updates are readily apparent. An analysis of these additional radar data provides strong, but not irrefutable, evidence that the anticyclonic tornado that may be inferred from WSR-88D data did not exist; consequently, upon discussions with the National Weather Service, it was not included in Storm Data.
\end{abstract}

\section{SIGNIFICANCE STATEMENT}

The official nationwide radar network in the United States is capable of detecting some tornadoes, but this capability generally decreases with increasing distance from the radar. In this study, we examined two peculiar radar signatures observed in a tornado-producing thunderstorm in Oklahoma in May 2016. These signatures looked similar to those seen in anticyclonic (i.e., clockwise-rotating in the Northern Hemisphere) tornadoes, but the location within the storm would have been highly unusual for a tornado. Data from a rapid-scan mobile radar closer to the storm revealed that the signatures were not from anticyclonic tornadoes but, rather, may have been quasi-horizontal vortices centered $\sim 1 \mathrm{~km}$ above ground. This case serves as an example of the benefits expected from a denser radar network of next-generation, rapid-scan weather radars.

\footnotetext{
${ }^{\text {a }}$ Current affiliation: Department of Civil and Environmental Engineering, University of Illinois at Urbana-Champaign, Urbana, Illinois.
}

Corresponding author: Jeffrey Snyder, jeffrey.snyder@noaa.gov 


\section{Introduction}

Despite several limitations, weather radars provide important storm-scale information to forecasters and researchers and have been used for more than half a century to study convective storms and tornadoes (e.g., Browning and Ludlam 1962; Atlas 1963; Lhermitte 1964; Burgess and Brown 1973; Lemon et al. 1977)—see Bluestein (2020) for a more complete review of this history. Owing to beam broadening and the limited number of weather radars in the United States (e.g., WSR-88D radars are generally spaced more than $200 \mathrm{~km}$ from one another), radar resolution from fixed-site radars is often too coarse to resolve the flow within tornadoes, although there may be a distinct tornadic vortex signature in radial velocity $V_{R}$ ( e.g., Donaldson 1970; Brown et al. 1978; Wood and Brown 1997; Brown and Wood 2012). In addition, because the center of a radar beam increases in altitude with increasing range in normal beam propagation environments on quasi-level terrain, the near-ground portion of almost all tornadoes is not sampled by fixed-site radars, save for the fortuitous close encounter (e.g., Burgess and Magsig 1998; Burgess et al. 2002). To improve detection, some have proposed a denser network of radars (e.g., McLaughlin et al. 2009), but such a system has not been built in the United States.

In contrast to fixed-site radars, mobile platforms can be used to bring radars closer to convective storms, which improves the probability of resolving tornadoes and sampling their near-ground flow structure. Even on scales larger than that of tornadoes [i.e., scales larger than $O(100) \mathrm{m}]$, improved resolution of a severe convective storm and more complete data coverage, particularly near the ground, provided by close-range radars can reduce data ambiguity and improve a user's ability to assess storm structure and tornado probability, structure, and intensity. For this reason, mobile weather radars have been a popular tool for observing and studying convective storms and tornadoes (e.g., Bluestein and Unruh 1989; Bluestein et al. 1995, 2007a, 2010; Wurman et al. 1997; Bluestein and Pazmany 2000; Biggerstaff et al. 2005; Weiss et al. 2009; Burgess et al. 2010; French et al. 2013; Isom et al. 2013; Pazmany et al. 2013; Wurman and Kosiba 2013; Kurdzo et al. 2017; Tanamachi et al. 2018).

This paper focuses on one of several supercells that together produced 12 tornadoes in Oklahoma on 9 May 2016 (Table 1 and Fig. 1). The particular supercell examined herein produced two tornadoes (A1 and A2 in Table 1) and was sampled by several WSR-88D radars. During the time period that serves as the focus of this study (i.e., $\sim 2145-2220$ UTC), the closest operational WSR-88D radar was $80-100 \mathrm{~km}$ away from the supercell of interest, though a nonoperational, ${ }^{1}$ research-focused WSR-88D radar was $\sim 10 \mathrm{~km}$ closer. At these ranges, the center of the radar beam at the lowest elevation angle (i.e., $0.5^{\circ}$ ) was approximately $1.2-1.3 \mathrm{~km}$ above ground level (AGL). Meanwhile, a rapid-scan, polarimetric mobile radar was collecting data much nearer to the supercell, which allowed it to collect data at much lower altitudes.

Shortly before 2150 UTC, the WSR-88D radars appeared to sample a strong anticyclonic circulation to the northeast of an ongoing cyclonic tornado, and a swath of damage was later identified by the Norman, Oklahoma, National Weather Service Forecast Office (hereafter OUN, not to be confused with the KOUN radar located in the same city). Herein, we will show, using the high-resolution data provided by the mobile radar, that the feature that was preliminarily thought to be an anticyclonic tornado as identified in the WSR-88D data was probably a (slightly tilted) quasi-horizontal vortex that passed northward and rearward through the forward flank of the supercell. Partly on account of some of the data presented herein that was shared with OUN, this event was not submitted to Storm Data as a tornado.

This case study is intended to provide a cautionary tale of needing to rely upon ${ }^{2}$ radar data collected $\sim O(100) \mathrm{km}$ away from a potentially tornadic supercell, something that operational meteorologists may need to do in the absence of other observations. Even though a tornado-like damage path (in terms of spatial orientation of a "path") was observed near the location of a couplet in data from the WSR-88D radars, a mobile radar located much closer to the storm was able to collect higher-resolution data with coverage significantly closer to the ground, and this latter dataset seemingly refutes the original supposition that an anticyclonic tornado moved northward through the forward flank echo region of the supercell. The additional data provided by this radar supports the argument that reducing

\footnotetext{
${ }^{1}$ We use "nonoperational" in the sense that the radar is not a part of the operational WSR-88D network in the United States, which comprises more than 150 radars that share a set of common volume coverage patterns (VCPs) and operate nearly $24 \mathrm{~h}$ a day every day of the year. This particular nonoperational radar often operates using customized, very atypical scanning strategies (to include sectors and range-height indicator scans) and tends to have extended down periods for hardware or software modifications.

${ }^{2}$ We do not use "rely upon" in a disparaging manner. Operational meteorologists use as many tools as are available, and radar data may be (and, along with visual/in-person reports, often is) the best data available from which to assess convective storm processes, structures, and threats.
} 
TABLE 1. A list of tornadoes that occurred in Oklahoma on the afternoon and evening (local time) of 9 May 2016. Tornadoes F2, F3, and F4 occurred early on 10 May 2016 when expressed in UTC. These data were collected from the Storm Events Database maintained by the National Centers for Environmental Information (NCEI). Lengths and widths have been converted from the units used in the database (i.e., miles and yards). The "EFU" rating represents an unknown EF-scale rating (typically provided when a dearth of damage indicators exist that make it extraordinarily difficult to assess intensity).

\begin{tabular}{ccccc}
\hline \hline ID No. & $\begin{array}{c}\text { Time } \\
(\text { UTC) }\end{array}$ & $\begin{array}{c}\text { EF-scale } \\
\text { rating }\end{array}$ & $\begin{array}{c}\text { Length } \\
(\mathrm{km})\end{array}$ & $\begin{array}{c}\text { Max } \\
\text { width }(\mathrm{m})\end{array}$ \\
\hline A1 & $2106-2127$ & EF4 & 14.5 & 366 \\
A2 & $2134-2217$ & EF3 & 27.4 & 1372 \\
B1 & $2216-2216$ & EFU & 0.2 & 9 \\
C1 & $2218-2225$ & EFU & 2.4 & 37 \\
D1 & $2218-2234$ & EF3 & 14.5 & 640 \\
D2 & $2246-2319$ & EF1 & 19.3 & 823 \\
D3 & $2247-2247$ & EFU & 0.8 & 23 \\
E1 & $2258-2319$ & EF2 & 9.7 & 549 \\
F1 & $2322-2342$ & EF3 & 22.2 & 2835 \\
F2 & $0002-0010$ & EF1 & 9.8 & 594 \\
F3 & $0018-0030$ & EFU & 9.7 & 137 \\
F4 & $0025-0026$ & EFU & 0.8 & 69 \\
\hline
\end{tabular}

between-radar distances can improve feature detection in severe convective storms owing to better spatial resolution and more complete low-level coverage at shorter ranges. In many cases, the desire to reduce the range between the radar and convective storm (or tornado or other feature of interest) is argued from the perspective of wanting to identify where hazardous weather phenomena (e.g., tornadoes) did occur, but, in this case, the closer distance and better low-level data coverage is used to show that a tornado likely did not occur.

Although the mobile radar data will be used to highlight the importance of high-resolution, near-ground data, we will also make use of a research-focused WSR-88D (KOUN) that was collecting data only over a $\sim 90^{\circ}$ sector in order to simulate the faster volume update times that may be provided by a next generation, phased-array-based radar system (e.g., Weber et al. 2007; Heinselman and Torres 2011). Improved temporal resolution can be expected to better capture the evolution of rapidly changing phenomena (e.g., Heinselman et al. 2008; Kuster et al. 2016), with downstream impacts on severe weather warning performance (Heinselman et al. 2015; Kuster et al. 2015; Wilson et al. 2017a). The use of KOUN in this case provides additional insight into the evolution and vertical structure of the suspect anticyclonic velocity couplet.

An overview of the radar and data used herein is presented in the following section. A brief discussion of the mesoscale environment and general evolution of convective storms on 9 May 2016 follows in section 3. A more detailed examination of the radial velocity $V_{R}$ anomalies is shown in section 4 , followed in section 5 by a discussion of our best hypothesis for the underlying features responsible for the $V_{R}$ anomalies seen in the radar data.

\section{Tools and data}

The radar data used in this study were collected by three polarimetric radars (Table 2). KTLX is an operational

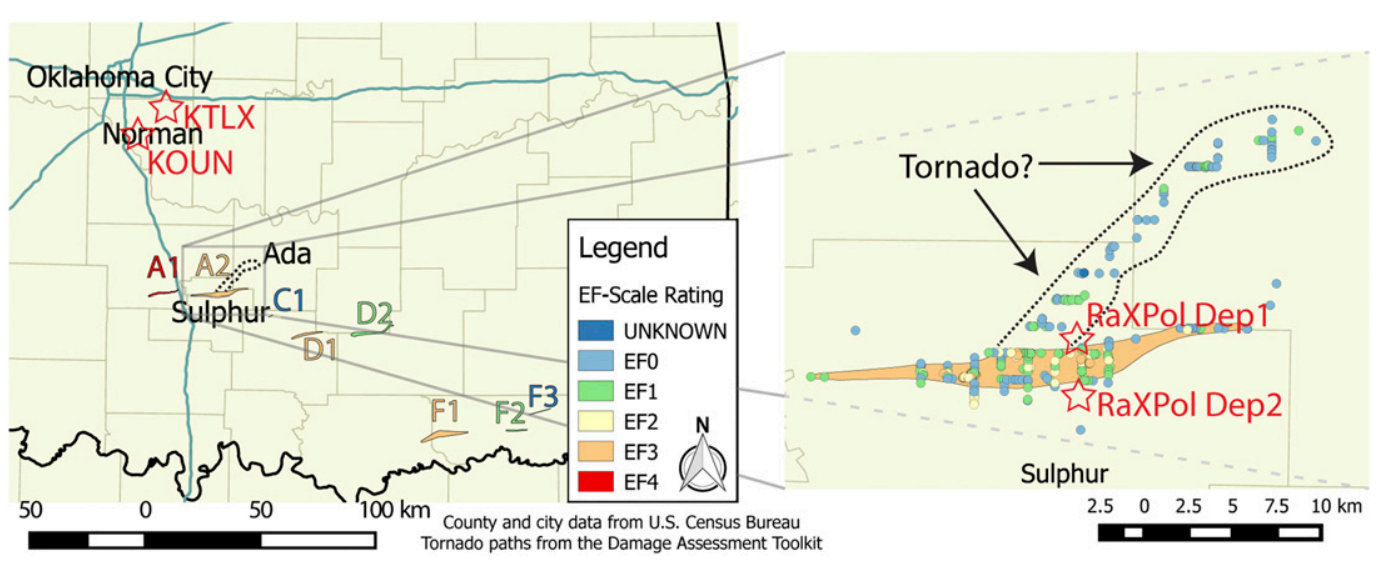

FIG. 1. A map of the paths of several tornadoes that occurred in southern Oklahoma on 9 May 2016. The labels adjacent to each tornado correspond to those in Table 1. Tornadoes B1 (in Norman), D3 (very near D2), and F4 (very near F3) were short lived, had very short paths less than $1.0 \mathrm{~km}$, and are not shown in order to minimize clutter. Tornado E1 occurred in northern Oklahoma outside the extent of this map. The inset panel on the right shows a "zoomed in" view of the path of the Sulphur tornado (A2). The black dotted area indicates an apparent "path" of damage that will serve as the focus of this paper. The red stars indicate the positions of the radars used herein. In the inset panel, RaXPol Dep1 and RaXPol Dep2 refer to RaXPol's first and second deployment locations. Dots represent damage indicators as recorded by the NWSFO OUN damage survey. The fill color for each path and dot represent the EF-scale rating assigned to the path or damage indicator. 
TABLE 2. Selected characteristics of the three radars from which data are used in this study. RaXPol's characteristics are specific to the configuration(s) used on 9 May 2016, as the range resolution, gate spacing, and rotation rate can be modified by the operator. The update intervals for KTLX and KOUN are specific to the time period between $\sim 2140$ and 2210 UTC. After accounting for beam smearing, the effective beamwidth of RaXPol, considering the dwell time and rotation rate, was closer to $1.4^{\circ}$. Since KOUN was collecting data over only a $\sim 90^{\circ}$ sector, volume update intervals were much shorter than they were from KTLX. KTLX's additional three scans at $0.5^{\circ}$ elevation angle provided by the use of MESO-SAILS (Chrisman 2014a,b) increased the frequency of lowest-level scans such that it was comparable to that from KOUN, but the use of SAILS came at the expense of an increase in volume collection time (i.e., decrease in the frequency of all other elevation angles). More details on the capabilities of RaXPol can be found in Pazmany et al. (2013). Beamwidths for KTLX and KOUN are from Ice et al. (2011).

\begin{tabular}{lccc}
\hline \hline & KTLX & KOUN & RaXPol \\
\hline Frequency (wavelength) & $2.910 \mathrm{GHz}(\sim 10.3 \mathrm{~cm})$ & $2.705 \mathrm{GHz}(\sim 11.1 \mathrm{~cm})$ & $9.730 \mathrm{GHz}+/-20 \mathrm{MHz}(\sim 3.1 \mathrm{~cm})$ \\
3-dB beamwidth & $0.91^{\circ}$ & $0.97^{\circ}$ & $1.0^{\circ}$ \\
Range resolution & $250 \mathrm{~m}$ & $250 \mathrm{~m}$ & $75 \mathrm{~m}$ \\
Range gate spacing & $250 \mathrm{~m}$ & $250 \mathrm{~m}$ & $30 \mathrm{~m}$ \\
Max rotation rate & $16^{\circ} \mathrm{s}-1$ & $30^{\circ} \mathrm{s}$ & $\sim 80^{-1} \mathrm{~s}^{-1}$ \\
Average volume update interval & $\sim 388 \mathrm{~s}$ & $\sim 93 \mathrm{~s}$ & $\sim 24 \mathrm{~s}$ \\
Average lowest elevation angle revisit & $\sim 96 \mathrm{~s}$ & $\sim 93 \mathrm{~s}$ & $\sim 24 \mathrm{~s}$ \\
$\quad$ interval & & & \\
\hline
\end{tabular}

WSR-88D radar located in southeastern Oklahoma City, Oklahoma, and KOUN is a research-focused WSR$88 \mathrm{D}$ radar located in Norman, Oklahoma. The former is part of the operational network of WSR-88D radars in the United States. The latter, currently owned and operated by the National Severe Storms Laboratory, is used for, among other purposes, studying severe convective storms in central Oklahoma with greater temporal resolution and customized scanning strategies that improve data coverage and data quality (e.g., Scharfenberg et al. 2005; Kumjian et al. 2010; Snyder and Ryzhkov 2015; Kuster et al. 2016, 2019; Tanamachi and Heinselman 2016; Witt et al. 2018). The data from KTLX, like all operational WSR-88D data, are generally always available to forecasters, media, and the public, whereas data from KOUN are seldom available to the public on a consistent basis and may or may not be available to others depending upon how the radar is being used.

The third radar used in this study is a mobile, rapidscan, polarimetric weather radar (RaXPol; Pazmany et al. 2013) owned and operated by the University of Oklahoma. To improve clarity, RaXPol's $V_{R}$ data have been filtered to remove areas of very low signal-to-noise ratio (i.e., SNR $<0 \mathrm{~dB}$ ) and low normalized coherent power (i.e., NCP $<0.20$ ). Although the effects of attenuation in mobile, $\mathrm{X}$-band radar data can be estimated provided a set of assumptions (e.g., Snyder et al. 2010), accurately estimating attenuation (and differential attenuation) through melting hail of unknown size and meltwater distribution can be difficult. In addition, there are some indications of debris fallout (e.g., Van Den Broeke 2015), which would further complicate attempts to accurately estimate and correct for attenuation. Because the features that serve as the focus of this paper are best seen in $V_{R}$ data, we do not attempt to correct for attenuation and differential attenuation in the data presented herein.

Data from the Oklahoma Climatological Survey's (OCS's) Oklahoma Mesonet (Brock et al. 1995) were used to examine the near-ground meteorological conditions and surface boundaries that may have affected the supercell examined in this paper, and the WeatherScope program (Wolfinbarger et al. 2004) was used to display the Mesonet data. Satellite data were obtained from NOAA's Comprehensive Large Array-data Stewardship System (CLASS) and NASA's Level-1 and Atmosphere Archive and Distribution System (LAADS) Distributed Active Archive Center (DAAC). Maps and the Visible Infrared Imaging Radiometer Suite (VIIRS) satellite imagery were plotted using QGIS v3.6.0. The HighResolution Rapid Refresh (HRRR) model data, limited as its use is in this study, were obtained from NOAA's Air Resource Laboratory data archive at https://www.ready.noaa.gov/READYamet.php. Finally, the Weather Decision Support System-Integrated Information (WDSS-II; Lakshmanan et al. 2007) software package was used to process the Level II WSR-88D data, automatically dealias the $V_{R}$ data, and interrogate the radar data.

\section{Meteorological and event overview}

On the afternoon of 9 May 2016, a dryline was located just west of Interstate Highway 35 in Oklahoma (Fig. 2a), and it was along this dryline and in the robust low-level moisture to its east that storms developed (Fig. 2b). A remnant outflow boundary, likely associated with convection that had occurred earlier in the day, was located in southern Oklahoma, with cooler air north of this boundary. Destabilization during the day resulted in 


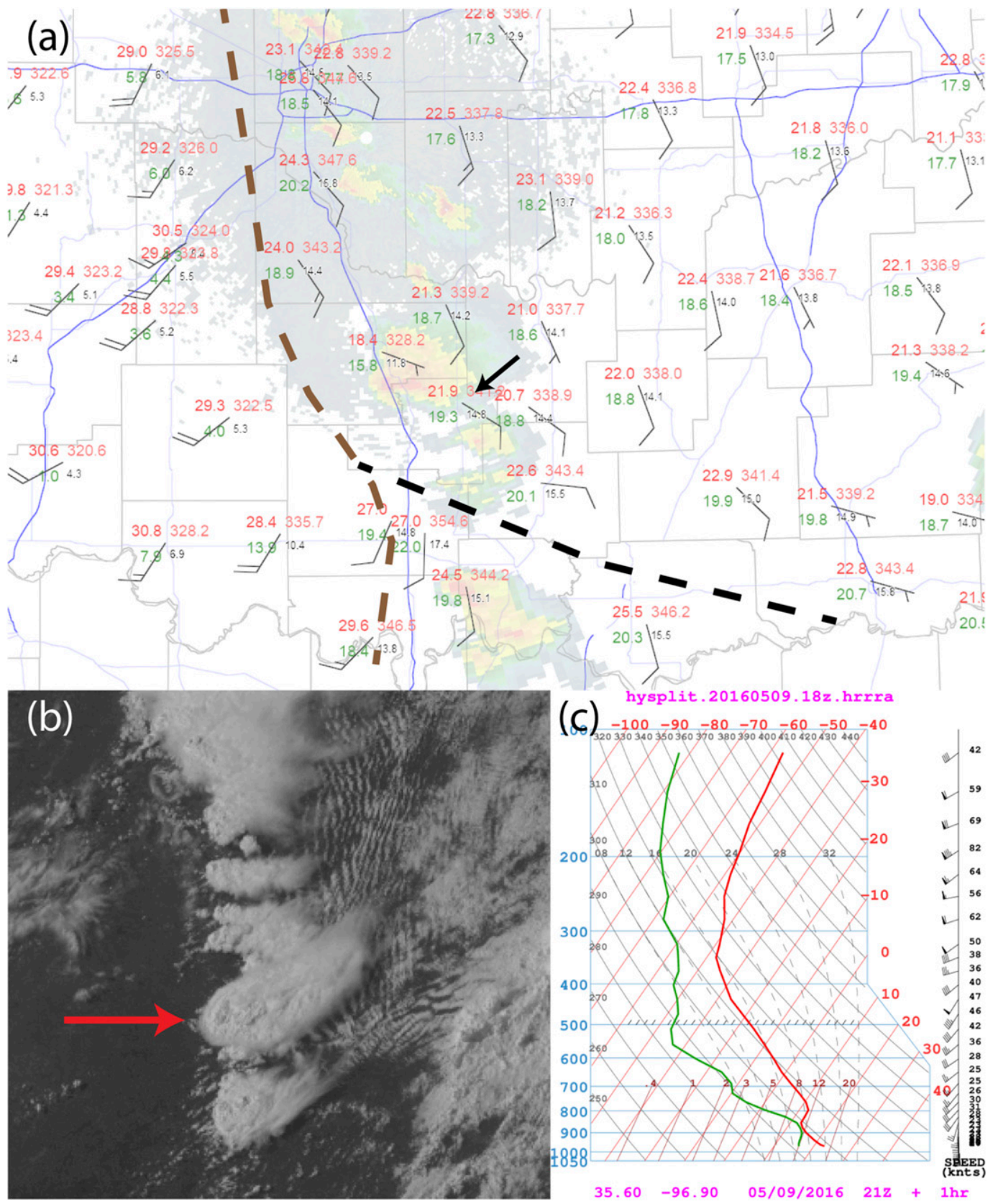

FIG. 2. Midafternoon meteorological data on 9 May 2016. (a) Surface observations from the Oklahoma Mesonet valid at 2130 UTC. Each observation location shows temperature (red; upper-left number; ${ }^{\circ} \mathrm{C}$ ), dewpoint temperature (green; lower-left number; ${ }^{\circ} \mathrm{C}$ ), equivalent potential temperature (light red; upper-right number; K), water vapor mixing ratio (black; lower-right number; $\mathrm{g} \mathrm{kg}^{-1}$ ), and $10 \mathrm{~m} \mathrm{AGL}$ wind speed (wind barb; $\mathrm{m} \mathrm{s}^{-1}$ ). The brown and black lines mark the approximate location of the dryline and outflow boundary, respectively. (b) Visible satellite image valid 2137 UTC from GOES-13 (NOAA/CLASS 2016). The red arrow points to the location of the Katy-Sulphur supercell. (c) HRRR forecast sounding northeast of Sulphur valid at 2100 UTC from the 1800 UTC model srun. The approximate location of this sounding is marked by the black arrow in (a).

moderate CAPE and weak CIN ahead of the dryline (not shown), except for an area of stronger capping in south-central and southeastern Oklahoma on the cool side of the outflow boundary. Paired with favorable low-level (e.g., $0-1$ and $0-3 \mathrm{~km}$ ) and deep-layer (e.g., $0-6 \mathrm{~km}$ ) wind profiles, the environment across Oklahoma to the east of the dryline, particularly near the outflow boundary where low-level shear was locally enhanced, 


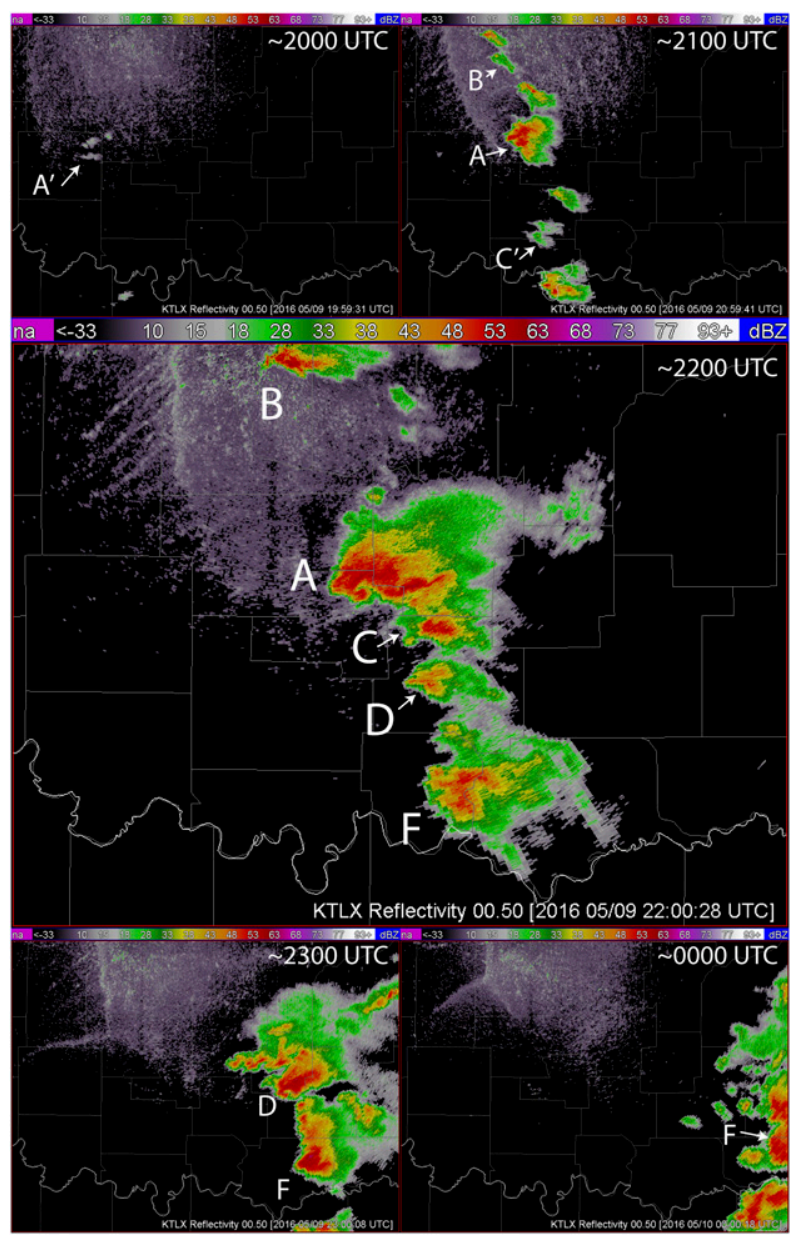

FIG. 3. An overview of the evolution of convective storms across southern Oklahoma on the afternoon of 9 May 2016 as seen using the $0.5^{\circ} Z_{H}$ data from KTLX (NOAA/NWS 1991). The time between each panel, as marked, is $\sim 1 \mathrm{~h}$. White letters adjacent to some of the convective storms denote parent storm that produced the tornado(es) as listed in Table 1 and shown in Fig. 1. A prime following a label indicates that the eventual tornadic storm arose from a complicated evolution associated with the (usually weak) radar echo. Supercell E occurred north of the area shown in this figure.

was supportive of tornadic supercells (Fig. 2c; e.g., Thompson et al. 2003).

The first robust convective storm of the event (hereafter referred to as the Katy-Sulphur supercell or Supercell A) developed near the dryline in south-central Oklahoma around 2000 UTC (Fig. 3). After producing a violent [EF4 on the enhanced Fujita scale (WSEC 2006)] tornado between 2106 and 2127 UTC $^{3}$ the supercell produced a second, larger tornado (commonly referred to as the "Sulphur"

\footnotetext{
${ }^{3}$ The start and end times used for the tornadoes in this manuscript are those listed in the National Centers for Environmental Information's Storm Events Database.
}

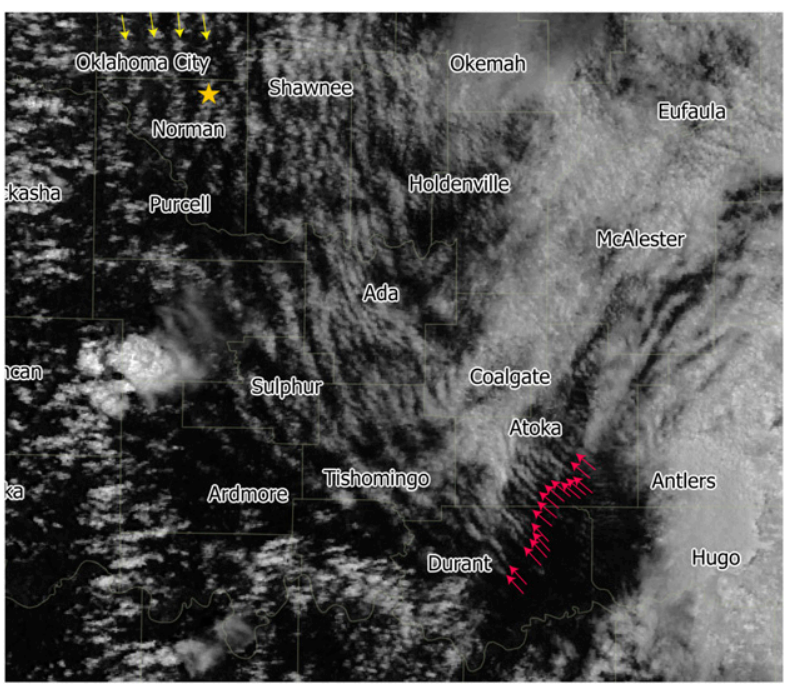

FIG. 4. A high-resolution visible satellite image (reflectance I1, $0.60-0.68 \mu \mathrm{m}$ ) obtained by VIIRS on the Suomi NPP satellite at $\sim 2006$ UTC. The yellow star southeast of the Oklahoma City label marks the location of KTLX. The red arrows near the lower-right part of the image point to several of the more closely space cloud streets in southeastern Oklahoma; the yellow arrows in the upperleft part of the image point to the more distantly spaced (and more meridionally oriented) cloud streets in central Oklahoma.

tornado and listed as A2 in Table 1) northwest of Sulphur, Oklahoma. This tornado moved generally eastward, producing damage as intense as EF3 and reaching a maximum width of $\sim 1370 \mathrm{~m}$ (1500 yards). This tornado dissipated at 2217 UTC.

Other supercells developed to the south and southeast of the Katy-Sulphur (A) supercell (Burgess et al. 2016). Destructive storm interactions, in addition to the possibility that the supercell was moving into the more highly capped environment in southeastern Oklahoma, weakened the Katy-Sulphur (A) supercell, and it did not produce any more tornadoes after $\sim 2217$ UTC.

Cloud streets were evident across eastern Oklahoma in visible satellite imagery during the afternoon as seen in GOES-13 data at $\sim 2130$ UTC (Fig. 2b). Higherresolution data from VIIRS on the Suomi National Polar-Orbiting Partnership (Suomi NPP) satellite from 2006 UTC more clearly show the structure of the curved banded clouds that resided east of an arc from Purcell to south of Sulphur to near Durant (Fig. 4). Similarly oriented bands of enhanced (but still low) equivalent radar reflectivity factor $Z_{H}$ are evident in KTLX data, particularly around 2100 UTC (Fig. 3). The cloud bands were more laminar in appearance, more closely spaced, and oriented in a southeast-northwest direction in southern Oklahoma near the outflow boundary (red arrows in Fig. 4) compared to those in central Oklahoma (which were less laminar, spaced farther apart, 
TABLE 3. The scanning strategies used by KTLX, KOUN, and RaXPol on 9 May 2016. Deployment information is also shown for RaXPol. The asterisks in the elevation angles provided for KTLX's VCP indicate angles that deviated very slightly from those defined by VCP 212 [i.e., Table 4-1 in OFCM (2016)]. In particular, VCP 212 calls for an elevation angle of $6.4^{\circ}$, whereas KTLX tended to scan at $\sim 6.45-6.47^{\circ}$ (which rounds to $6.5^{\circ}$ ), and $10.0^{\circ}$, whereas KTLX tended to scan at $\sim 10.05^{\circ}-10.06^{\circ}$ (which rounds to $10.1^{\circ}$ ), during the period examined in this paper.

\begin{tabular}{lcc}
\hline \hline & Time (UTC) & Elevation angles \\
\hline KTLX & & \\
VCP 212 with MESO-SAILSx3 & Event duration & $0.5^{\circ}, 0.9^{\circ}, 1.3^{\circ}, 1.8^{\circ}, 2.4^{\circ}, 3.1^{\circ}, 4.0^{\circ}, 5.1^{\circ}, 6.4^{\circ}, 0^{\circ}, 0^{\circ}$, \\
& & $10.0^{\circ}, 12.5^{\circ}, 15.6^{\circ}, 19.5^{\circ}$ \\
KOUN & $1919-2022$ & $0.5^{\circ}, 0.9^{\circ}, 1.4^{\circ}, 2.0^{\circ}, 2.7^{\circ}, 3.5^{\circ}, 4.4^{\circ}, 5.5^{\circ}, 6.5^{\circ}, 7.7^{\circ}$ \\
Custom VCP 1 & $2024-2209$ & $0.5^{\circ}, 0.9^{\circ}, 1.4^{\circ}, 2.4^{\circ}, 3.5^{\circ}, 4.6^{\circ}, 5.7^{\circ}, 7.1^{\circ}, 9.1^{\circ}, 11.4^{\circ}$ \\
Custom VCP 2 & $2210-2323$ & $0.5^{\circ}, 0.9^{\circ}, 1.4^{\circ}, 2.0^{\circ}, 2.7^{\circ}, 3.5^{\circ}, 4.4^{\circ}, 5.5^{\circ}, 6.5^{\circ}, 7.7^{\circ}$ \\
Custom VCP 1 & \\
RaXPol & $2143-2150$ & $0^{\circ}-20^{\circ}$ every $2^{\circ}$ \\
Custom VCP T1 (deployment No. 1) & $2151-2156$ & $0^{\circ}-10^{\circ}$ every $1^{\circ}$ \\
Custom VCP T2 (deployment No. 1) & $2156-2157$ & $0^{\circ}-10^{\circ}$ every $1^{\circ}$ \\
Custom VCP T2 (in motion) & $2159-2229$ & $0^{\circ}-10^{\circ}$ every $1^{\circ}$ \\
Custom VCP T2 (deployment No. 2) & $2230-2231$ & $0^{\circ}-20^{\circ}$ every $2^{\circ}$ \\
Custom VCP T1 (deployment No. 2) & & \\
\hline
\end{tabular}

and generally meridionally oriented as highlighted by the yellow arrows in Fig. 4). It is presumably not a coincidence that the surface winds in southeastern Oklahoma were generally more backed from the eastsoutheast while winds in central Oklahoma were generally from the southeast or south-southeast, and the difference in the orientation of the cloud streets is likely indicative of spatial variations in the boundary layer wind profile. These bands, which generally were stationary or moved slowly to the west with time, are probably a visual manifestation of horizontal convective rolls (HCRs; e.g., Weckwerth et al. 1997, 1999). Although the formation mechanisms of HCRs are not entirely understood, their presence indicates the existence of a capping layer atop the convective boundary layer (e.g., Christian and Wakimoto 1989) and static stability (e.g., Scofield and Purdom 1986; Bluestein and Snyder 2015), which would be consistent with the presence of cooler near-ground air on the north side of the outflow boundary and the associated stable layer that begins near $\sim 1-1.5 \mathrm{~km}$ AGL (e.g., Fig. 2c). Although HCRs can affect convective storm initiation and evolution (e.g., Thompson and Edwards 2000), more detailed radar analysis of the HCRs on this day closer to the outflow boundary in southern Oklahoma is not possible owing to sensitivity and beam height limitations.

RaXPol targeted the Katy-Sulphur (A) supercell while the storm was producing the Sulphur (A2) tornado. The radar began collecting data at 2142 UTC from a position $\sim 7.3 \mathrm{~km}$ north of Sulphur. Owing to the approach of the large tornado, the radar was relocated approximately $3.3 \mathrm{~km}$ southward between $\sim 2154$ and 2159 UTC. The maximum elevation angle scanned was either $10^{\circ}$ or $20^{\circ}$ depending upon the specific time. More details on the scanning strategies and deployments are presented in Table 3.

Around 2146 UTC, while the Sulphur tornado (A2) was ongoing, an anticyclonic couplet appeared in data from KTLX (Fig. 5). The couplet was also evident in KOUN data, though these data were not available to meteorologists in real time. At the time this couplet first appeared, it was located to the east or northeast of the primary cyclonic tornado. The couplet moved generally northward.

Damage near the path of this couplet was not reported to the NWS during or after the time during which the couplet was observed until a survey team from OUN documented the damage. The survey team identified a southwest-northeast-oriented band of intermittent damage and preliminarily indicated that the damage, which occurred very near the location of the anticyclonic couplet observed by KTLX, may have been associated with an anticyclonic tornado (G. Garfield and R. Prentice 2016, personal communication). The zone of damage was $\sim 1-2 \mathrm{~km}$ wide and $\sim 20-25 \mathrm{~km}$ long, and the maximum EF-scale rating assigned to the damage was EF1. The damage indicators were primarily trees, outbuildings, manufactured homes, and one- and two-family residences. Importantly, however, owing to limited time and very limited opportunities to talk to residents in the area, the surveyors were unable to establish the time at which the damage occurred or any first-hand oral or written reports or experiences from those whose property was damaged. Reports from the surveyors and photographs of the damage do not appear to show a strong signal for convergence or rotation, though the density of damage indicators was low in places. Nonetheless, the geometry of the damage path is consistent with that 


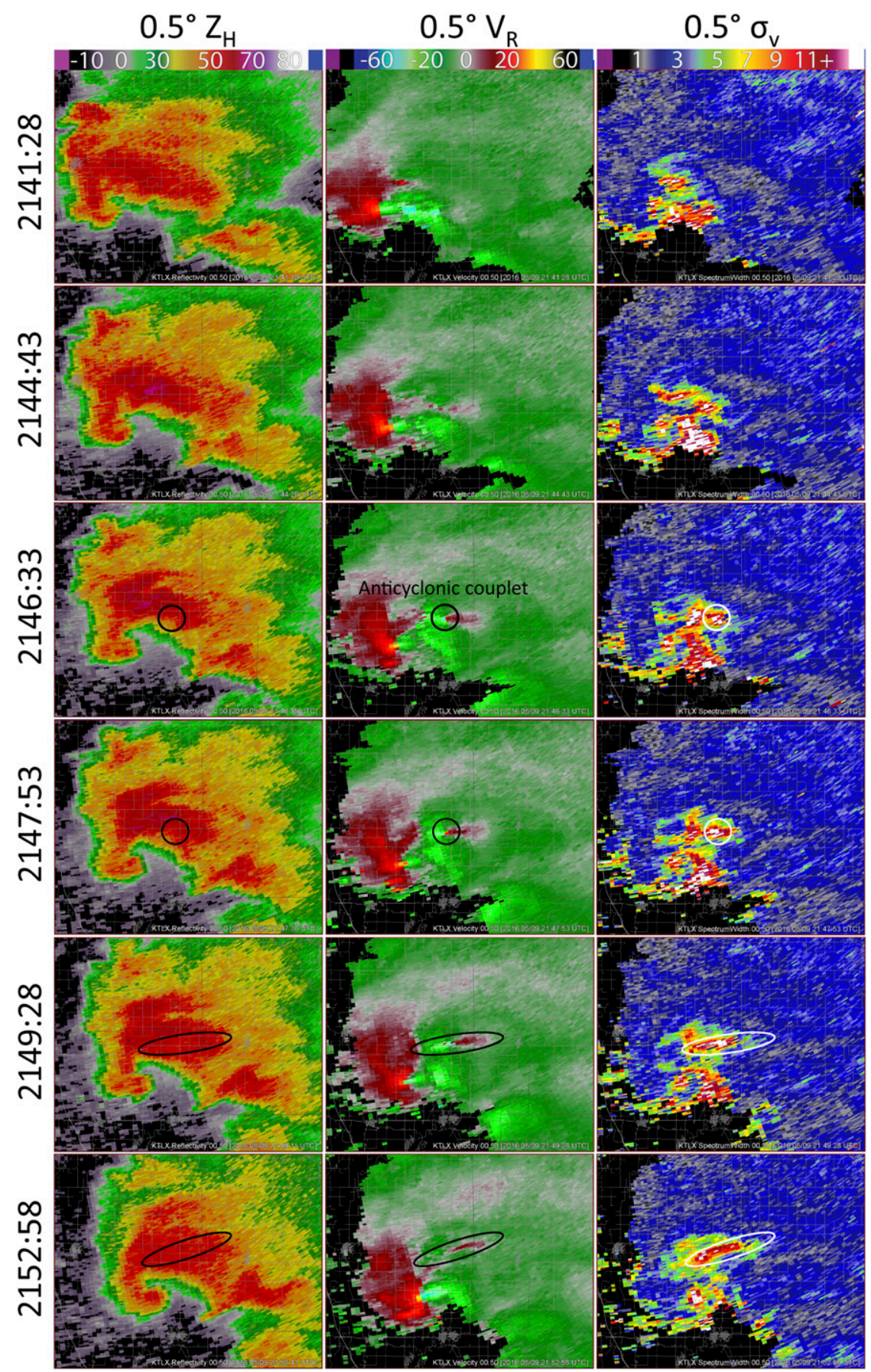

FIG. 5. (left) $Z_{H}$, (center) $V_{R}$, and (right) $\sigma_{v}$ at six selected times from KTLX. All data are from the $0.5^{\circ}$ elevation angle scan. Black (or white, in the case of those in the right column) circles and ellipses track the location of the anticyclonic vortex signature or shear zone. The radar is located to the northnorthwest of the storm, so inbound velocities (green) represent winds with a south-southeast component, whereas outbound velocities (red) represent winds with a north-northwest component.

usually associated with tornadoes (i.e., comparatively long and narrow).

In an attempt to learn more about the extent and nature of the damage corresponding to the in-person survey, we examined poststorm high-resolution, multispectral Landsat 8 and Sentinel-2 data (e.g., Yuan et al. 2002; Jedlovec et al. 2006; Molthan et al. 2014; Kingfield and de Beurs 2017). Unfortunately, abundant cloudiness 
in the first 4-6 weeks following the event and the sparse nature of trees in the areas damaged limited what we could learn about the extent and nature of the damage, and we did not use these data in our analysis as a result. Additionally, while Google Earth aerial imagery allowed us to see some of the tree damage that was reported during the damage survey (almost all of which appeared to be caused by westerly, southwesterly, and southerly winds), the large (3 year) gap in the imagery did not make this source worthwhile for our use.

Although not particularly common, anticyclonic tornadoes are occasionally observed in right-moving supercells (e.g., Brown and Knupp 1980; Wurman and Kosiba 2013). Bluestein et al. (2016) studied several cyclonic-anticyclonic tornado pairs in radar-observed supercells and found that the anticyclonic tornadoes were usually located to the south (or to the right when forward is defined as being along the storm motion vector) of the primary cyclonic tornado associated with the cyclonic low-level mesocyclone. The main anticyclonic couplet in the Katy-Sulphur (A) supercell was downstream of the primary cyclonic tornado and moved northward (or to the left of the forward storm motion vector) further into the forward flank echo, an unusual location for a tornado given the general structure of most supercells (e.g., Lemon and Doswell 1979). The very atypical location and behavior of this apparent anticyclonic tornado motivated our investigation of this feature, the results of which are reported and discussed in the next two sections.

\section{Radar examination of the anticyclonic signatures}

\section{a. WSR-88D observations}

Shortly before the anticyclonic couplet appeared in the WSR-88D data, the tornadic supercell had a classic hook echo (e.g., Fujita 1958; Markowski 2002) and strong cyclonic couplet in radial velocity $V_{R}$ associated with the ongoing Sulphur (A2) tornado (Fig. 5). Around 2141 UTC, KTLX sampled a narrow band of outbound velocities on the lowest elevation angle $\left(0.5^{\circ}\right) 6-7 \mathrm{~km}$ northeast of the tornado. This feature, which moved generally northward and was otherwise unremarkable, was associated with high spectrum width $\sigma_{v}$ (up to $19 \mathrm{~m} \mathrm{~s}^{-1}$ ) and $Z_{H}$ of $40-60 \mathrm{~dB} Z$. At 2144:43 UTC, a narrow but longer east-west-oriented zone of weak outbound velocities is seen to the northeast of the tornado, though it is not immediately clear in these KTLX data whether this second band of weak outbound $V_{R}$ is indeed separate from the first. A similarly oriented band of enhanced $\sigma_{v}$ also developed in this area. By 2146: 33 UTC, there was an anticyclonic couplet along the eastern edge of this zonally oriented band of enhanced $\sigma_{v}$ near the southern edge of the forward-flank echo (denoted by a black circle in Fig. 5) as the band of weak outbounds was "split" by strong inbound $V_{R}$. By $\sim 2149$ : 28 UTC, the anticyclonic couplet, associated extrema in $V_{R}$ (outbounds on the east side and inbounds on the west side), and enhanced $\sigma_{v}$ had moved northward. The feature continued moving generally to the north and became much less apparent by 2152:58 UTC as gateto-gate shear and the magnitude of $\sigma_{v}$ decreased. The elongated anomaly in $V_{R}$ appeared to curve to the southwest into the western periphery of the tornadic circulation.

To the west of the anticyclonic couplet was a region of cyclonic shear; the cyclonic and anticyclonic shear areas or couplets straddled a north-south region of enhanced inbound $V_{R}$ (i.e., winds with a strong southerly or southeasterly component). In fact, the cyclonic shear to the west of the anticyclonic couplet looks to have been stronger (on a gate-to-gate basis) than the anticyclonic couplet at $0.9^{\circ}$ and $1.4^{\circ}$ elevations (not shown), though the anticyclonic couplet was stronger than the cyclonic couplet on the $0.5^{\circ}$ scan.

A (second) similar feature was evident in $V_{R}$ between $\sim 2157$ and 2210 UTC, though it was more apparent at $0.9^{\circ}$ and $1.4^{\circ}$ (Fig. 6). At $0.5^{\circ}$, the $V_{R}$ anomaly was primarily seen as a narrow, zonally oriented band of light outbounds separating larger areas of stronger inbounds. As in the previous "anomaly", this band of enhanced $V_{R}$ was associated with a pronounced enhancement of $\sigma_{v}$ exceeding $10-15 \mathrm{~m} \mathrm{~s}^{-1}$.

The approximate locations and orientations of these two $V_{R}$ anomalies are shown in Fig. 7. Both features began near the southern edge of the forward flank echo and generally moved northward or north-northeastward with time, deeper into the forward flank echo. The second anomaly rotated counterclockwise with time, such that, at the last time at which it was evident near 2210 UTC, it was generally oriented northeast-southwest. The first anomaly also rotated slightly counterclockwise with time, though to a much lesser extent relative to the second.

\section{b. RaXPol observations}

The first $V_{R}$ anomaly observed by KTLX and associated with the first anticyclonic couplet originated extremely close to RaXPol. When it was first seen in the WSR-88D data, the anticyclonic couplet was less than $2 \mathrm{~km}$ north of RaXPol; by $\sim 2149$ UTC, it was $\sim 3.5 \mathrm{~km}$ north of RaXPol. Consequently, even though RaXPol was scanning to $20^{\circ}$ in elevation, RaXPol did not sample to the same altitude as the WSR- $88 \mathrm{D}$ radars' $0.5^{\circ}$ elevation scan owing to the short distance between RaXPol and the couplet. However, RaXPol collected $\sim 8-10$ elevation 

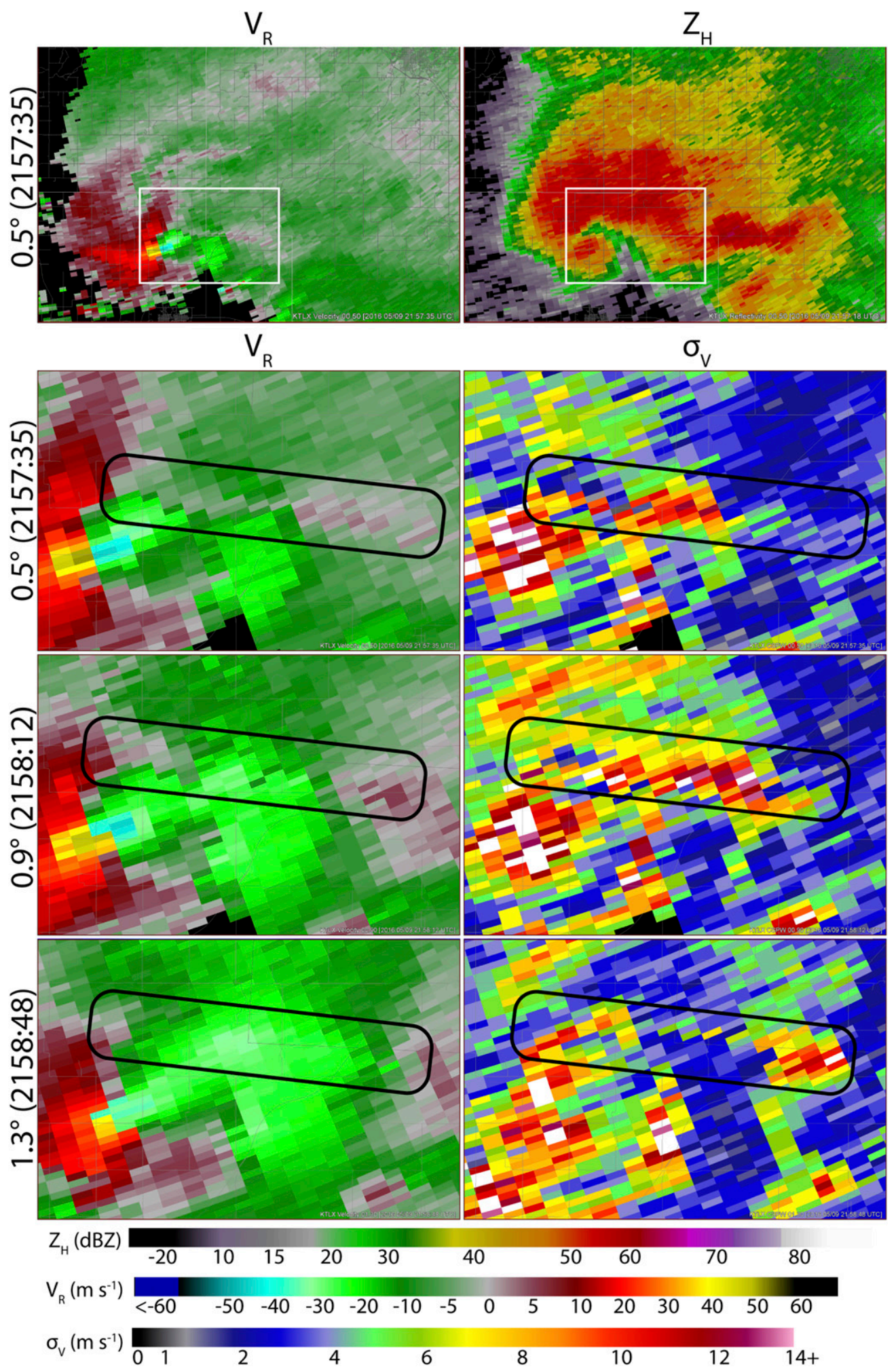

FIG. 6. KTLX data from the $0.5^{\circ}, 0.9^{\circ}$, and $1.3^{\circ}$ elevation angle scans revealing the vertical structure of the second $V_{R}$ feature/anomaly observed near 2158-2159 UTC. The top row provides a broader (i.e., storm-scale) perspective of (left) $V_{R}$ and (right) $Z_{H}$; the bottom three rows provide a zoomed in view of (left) $V_{R}$ and (right) $\sigma_{v}$, where the area shown is approximately that within the white rectangle provided in the top row of images. The black, rounded rectangles denote the locations of the second elongated velocity feature. 


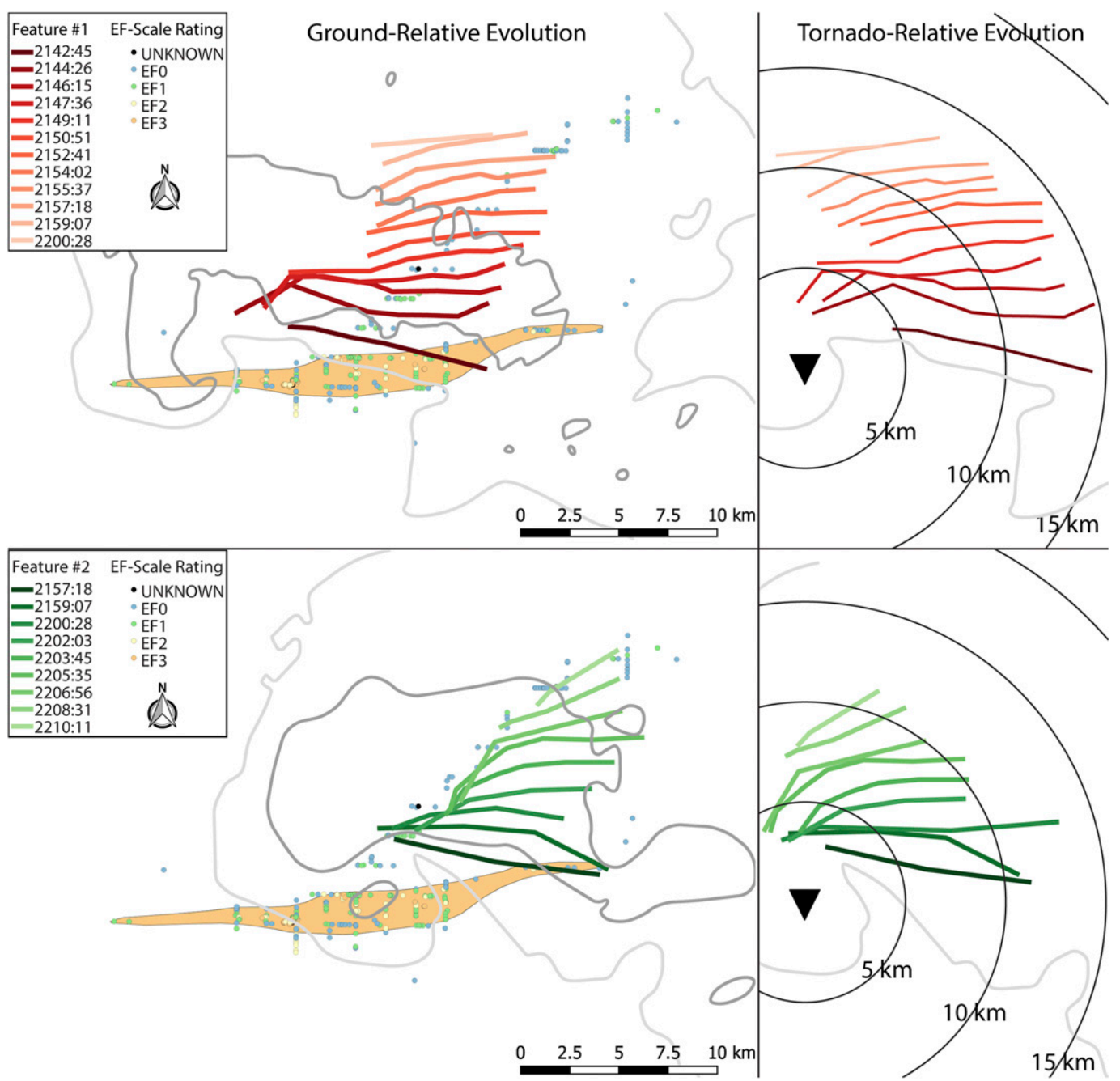

FIG. 7. Evolution of the two quasi-linear $V_{R}$ anomalies on the $0.5^{\circ}$ elevation angle scans from KTLX from (left) ground-relative and (right) tornado-relative reference frames. The first (top panel) is marked in red/orange, with the darkest color at 2142:45 UTC and the lightest at 2200:28 UTC; the second (bottom panel) is marked in green, with the darkest color at 2157:18 UTC and the lightest at 2210:11 UTC. The location of each anomaly is subjectively determined by the centerline of the anomaly or perturbation in the $V_{R}$ field. Colored dots represent the location of observed damage, and the orange swath marks the path of the cyclonic tornado according to the official damage survey. The light gray lines represent the approximate 30-dBZ isoecho at the start of each anomaly time (i.e., 2142 and 2157 UTC in the top and bottom panels, respectively). Additionally, the 50-dBZ isoecho is shown in dark gray in the left/ground-relative panels.

angles below the center of the lowest scans from KTLX and KOUN.

At the lower elevation angles from RaXPol, $V_{R}$ showed no tornadic vortex during the time and near the area in which KTLX and KOUN sampled the first elongated $V_{R}$ anomaly and anticyclonic couplet. From the time that RaXPol started collecting data near 2143 UTC, there was relatively little of note near the WSR-88D-identified anticyclonic couplet. Over the next several minutes, a zonally oriented band of inbound velocities (i.e., flow with a northerly component) developed and intensified in the lower elevation scans to the north of RaXPol (Fig. 8). Rather than an anticyclonic vortex couplet, the low-level RaXPol data suggest that a downburst developed in the supercell's forward flank during this time while the large, cyclonic tornado was occurring to the southwest (seen readily in $V_{R}$ ). The downburst signature can be seen well at $\sim 2150$ UTC, bounded by the orange oval in Fig. 9. A smaller cyclonic vortex was observed to the immediate southwest of this downburst and to the north of the main tornado, and a smaller anticyclonic couplet (or region of shear) was 


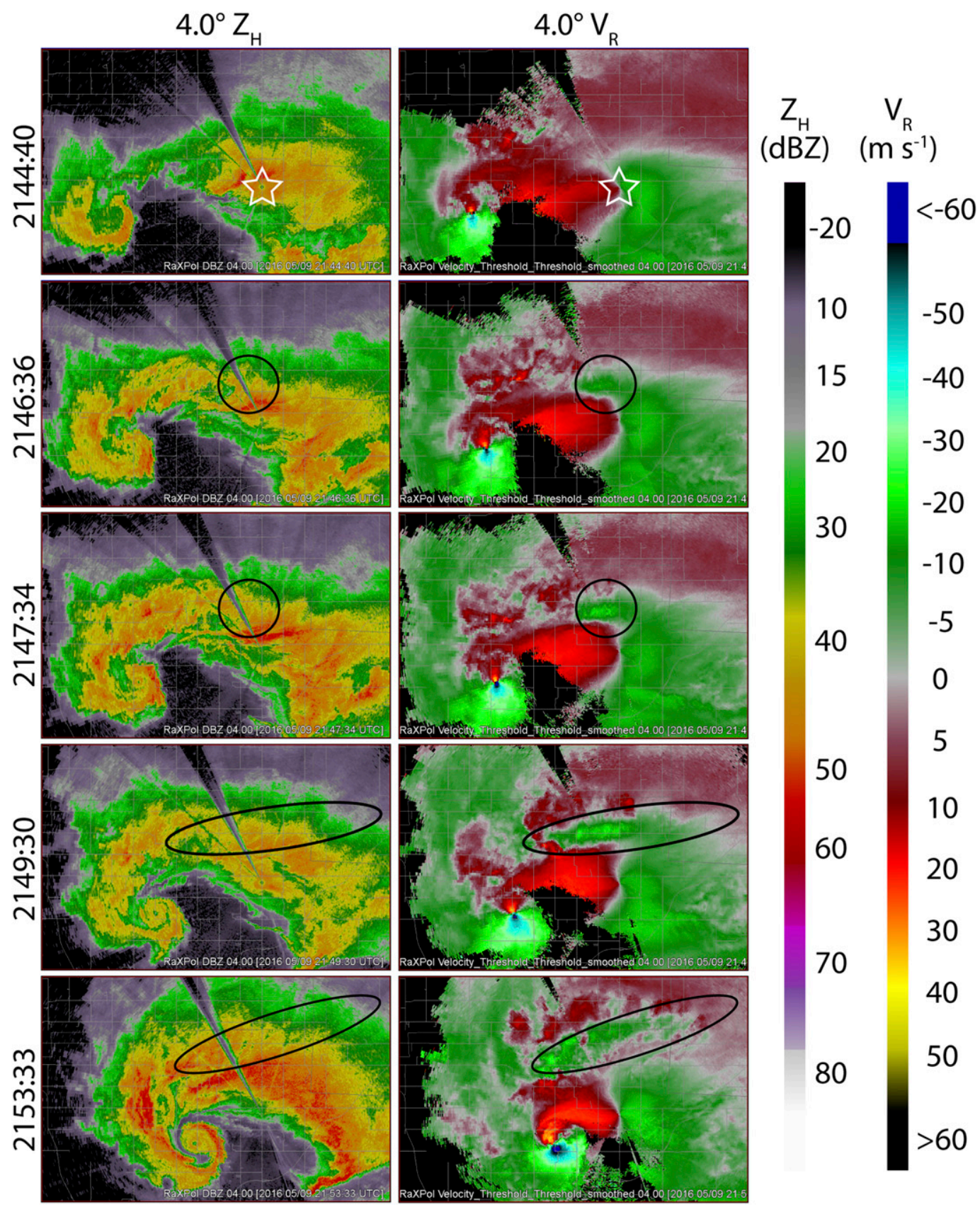

FIG. 8. A selected series of $4.0^{\circ}$ elevation angle scans from RaXPol with times similar to those in Fig. 5 showing (left) $Z_{H}$ and (right) $V_{R}$. A very narrow sector with partial beam blockage is evident to the north-northwest of the radar (the location of which is marked by a white star in the top panels). The black circles and ovals correspond to those provided in Fig. 5 and highlight the anticyclonic couplet and elongated $V_{R}$ anomaly observed by KTLX (and KOUN). The effects of attenuation (both from propagation through precipitation and a very wet radome) have not been estimated or removed.

apparent along the western periphery of the downburst. These couplets moved generally southwestward or southward toward the western edge of the large, cyclonic tornado.

Looking near the top of RaXPol's sampled volume provides data closer to the altitude at which the lower-level radar beams from the WSR-88D radars were likely located. Looking at RaXPol's higher elevations, an anticyclonic vortex signature was indeed apparent (Fig. 10). The times included in Fig. 10 are similar to those shown in Fig. 5, and the black circles and ovals in 


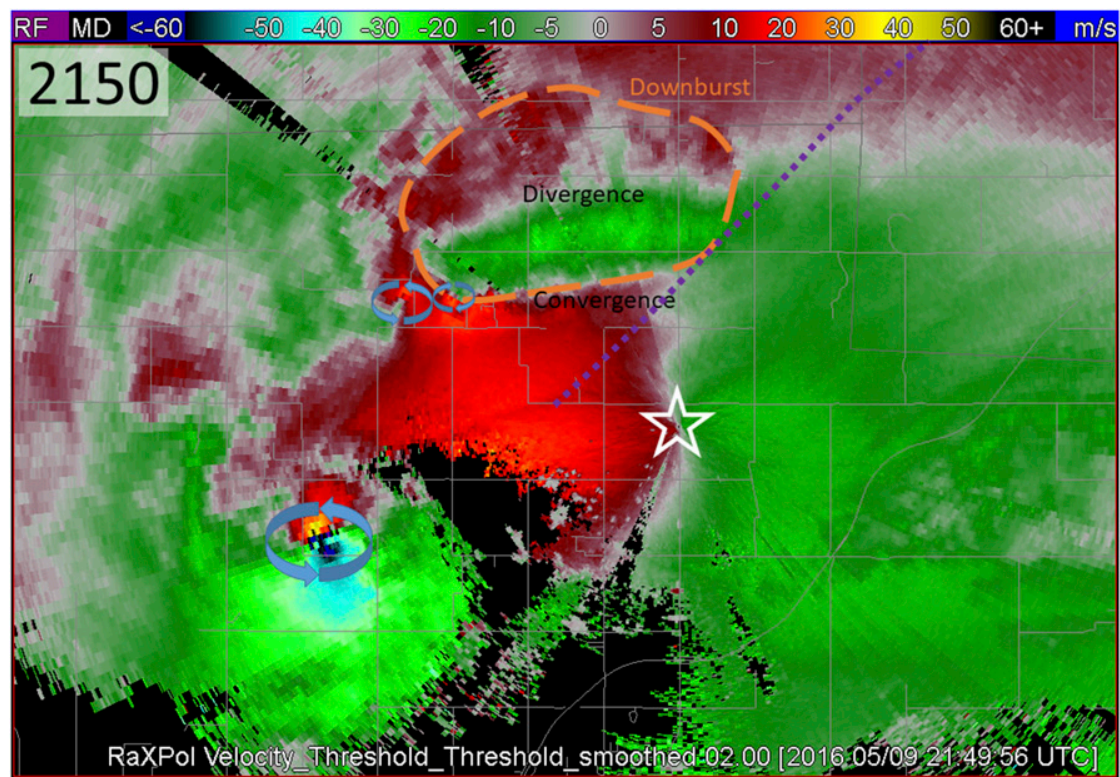

FIG. 9. RaXPol $2.0^{\circ}$ elevation angle $V_{R}$ data valid near 2150 UTC. The dashed orange oval highlights a divergence signature and probable downburst to the northeast of the tornado (which is marked by the large, blue, rounded arrows) and to the north-northwest of the radar. A smaller cyclonic vortex and even smaller anticyclonic vortex are apparent to the northnortheast of the main tornado, near the southwestern periphery of the divergence signature, and are marked by small blue circles. The approximate centerline of the damage swath is denoted by the dotted, purple line. RaXPol's location is marked by the white star.

Fig. 10 are the same as those in Fig. 5. By 2146: 36 UTC, a region of anticyclonic shear was located $\sim 3 \mathrm{~km}$ to the northwest of RaXPol, and this area of anticyclonic shear moved generally north or northeastward through 2150 UTC, after which time the scanning strategy was changed and such high elevation angles were no longer being collected. As in the KTLX and KOUN observations, the feature appeared to be associated with a zonally elongated band of enhanced $V_{R}$, particularly evident by $2149: 30$ UTC. Unlike in the KTLX and KOUN data, RaXPol measured a distinct (10-20 dBZ) reduction in $Z_{H}$ in the area of the anticyclonic shear and, at 2149:30 UTC, along a 3-4-km length of the elongated $V_{R}$ enhancement (white arrows in Fig. 10). As with the lower elevations (not shown), reduced copolar correlation coefficient $\rho_{\text {hv }}$ consistent with hail is apparent near RaXPol, though $\rho_{\mathrm{hv}}<0.80$ covers a larger area at this higher elevation angle, including along the narrow zone of reduced $Z_{H}$ at 2149:30 UTC.

As the strong tornado approached from the westsouthwest (Fig. 11), data collection was intermittent from $\sim 2155$ to 2158 UTC as the radar truck was driven southward to get out of the way of the approaching tornado. Stationary data collection began again near 2159 UTC, although the truck was not leveled until shortly after 2200 UTC. While unlevel, the pitch, roll, and orientation of the radar truck meant that the radar scanned higher than originally anticipated to the northeast through southeast of the truck, which was fortuitous because RaXPol was able to scan close to the altitude of the lowest scan from the WSR-88D radars even though RaXPol's highest (truck-relative) elevation angle was only $10.0^{\circ}$ at this time. Although no strong rotation was apparent in the forward-flank echo on the lower elevation scans, another anomalous, zonally oriented band of enhanced velocities in the rear (i.e., west and southwest portion) of the forward-flank echo to the northeast of the tornado was apparent at higher elevation angles around 2200 UTC (Fig. 12). At these higher elevation angles, the band of enhanced $V_{R}$ (primarily inbounds at $8.0^{\circ}$ and outbounds at $10.0^{\circ}$ ) was collocated with reductions in $Z_{H}$ and $\rho_{\mathrm{hv}}$ (dotted lines in Fig. 12) and a local enhancement in $\sigma_{v}$. In fact, at $8.0^{\circ}, Z_{H}$ dropped to $\sim 10 \mathrm{~dB} Z, \rho_{\mathrm{hv}}$ decreased to as low as $\sim 0.6$, and $\sigma_{v}$ increased to $6-12 \mathrm{~m} \mathrm{~s}^{-1}$. At $10.0^{\circ}$, reduced $Z_{H}$ and $\rho_{\mathrm{hv}}$ and enhanced $\sigma_{v}$ were primarily located to the west and east of the region of enhanced outbound velocities where there was cyclonic (on the west end) and anticyclonic (on the east end) shear, similar to the KTLX fields shown in Fig. 6. At $5.0^{\circ}$, a zonally oriented band of reduced $Z_{H}$ and $\rho_{\mathrm{hv}}$ can be seen, but there is little apparent signal in $V_{R}$ except for some weak radial convergence. 

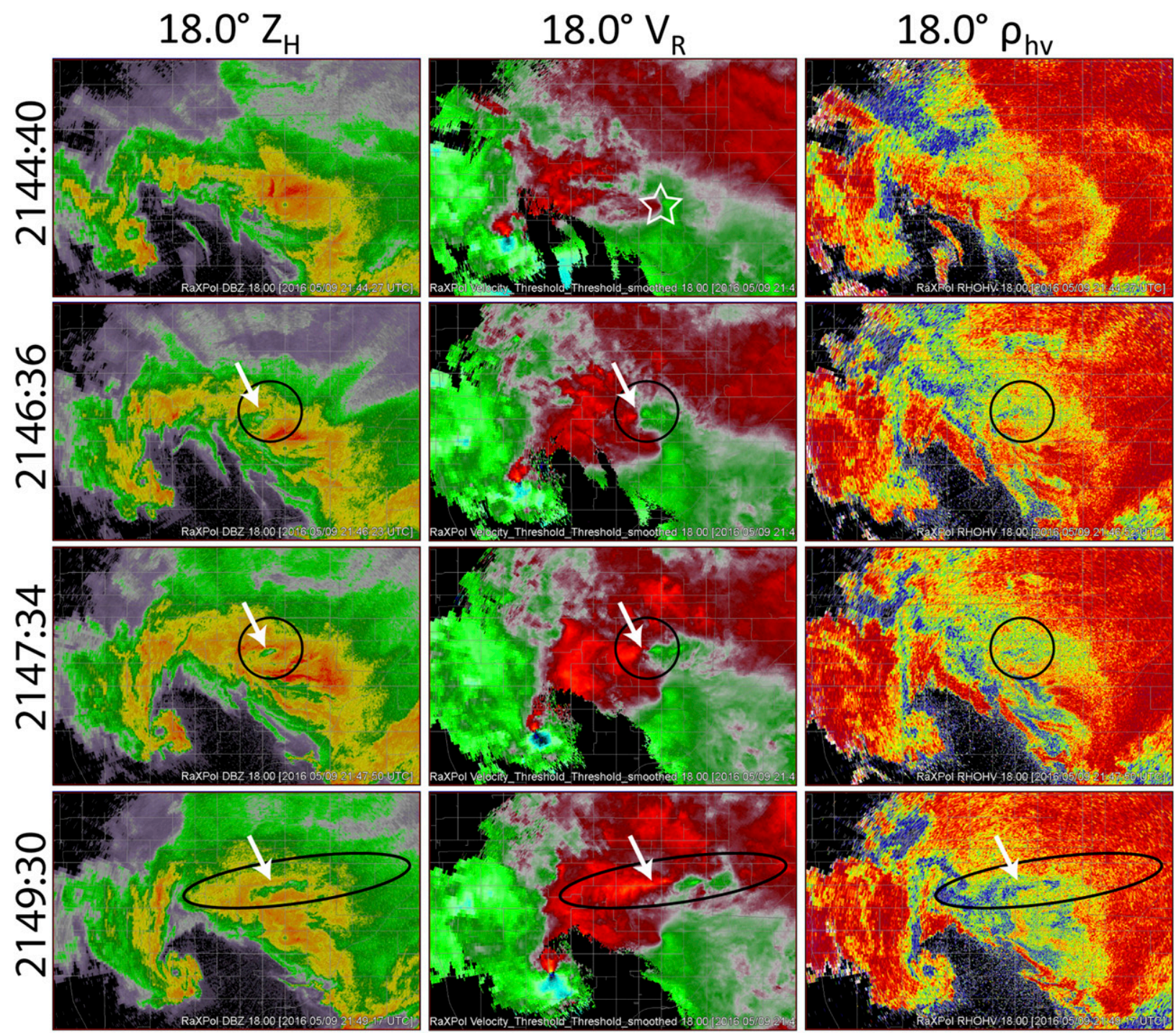

$$
\begin{aligned}
& Z_{H}(d B Z) \\
& V_{R}\left(m^{-1}\right) \\
& \rho_{H V}
\end{aligned}
$$$$
\begin{array}{lllll}
-20 & 10 & 15 & 20 & 30
\end{array}
$$$$
40
$$$$
50
$$$$
60
$$$$
70
$$
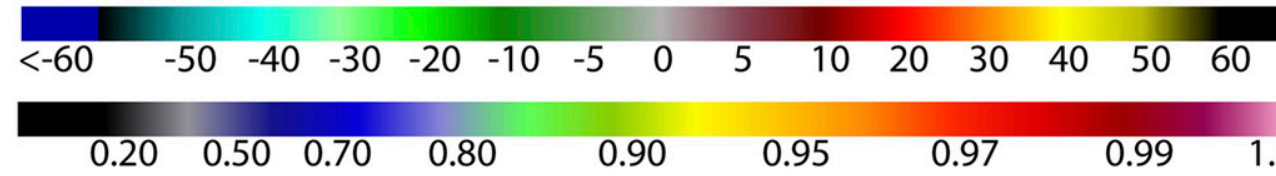

\subsection{0}

0.95

0.97

0.99

FIG. 10. As in Fig. 8, but from the $18.0^{\circ}$ elevation angle and including (right) $\rho_{\text {hv }}$. The white arrows highlight the anticyclonic couplet and (by 2149:30 UTC) elongated enhancement in outbound $V_{R}$. RaXPol's location is marked by the white star in the uppermost $V_{R}$ panel.

The very large tornadic debris signature associated with the ongoing cyclonic tornado (e.g., Ryzhkov et al. 2005; Bluestein et al. 2007a, 2015, 2018; Bodine et al. 2011, 2014; Bunkers and Baxter 2011; Schultz et al. 2012; Snyder and Bluestein 2014; Van Den Broeke and Jauernic 2014; Kurdzo et al. 2015; Van Den Broeke 2015; Wakimoto et al. 2015, 2016; Houser et al. 2016;
Griffin et al. 2017) is apparent to the immediate northwest of the radar.

For more granularity, looking at all elevation angles at and above $5.0^{\circ}$, we can see that the anomaly was characterized by enhanced inbound velocities on the $6.0^{\circ}$ and $7.0^{\circ}$ elevation angles that transitioned to primarily outbound velocities at $9.0^{\circ}$ and $10.0^{\circ}$ elevation angles 


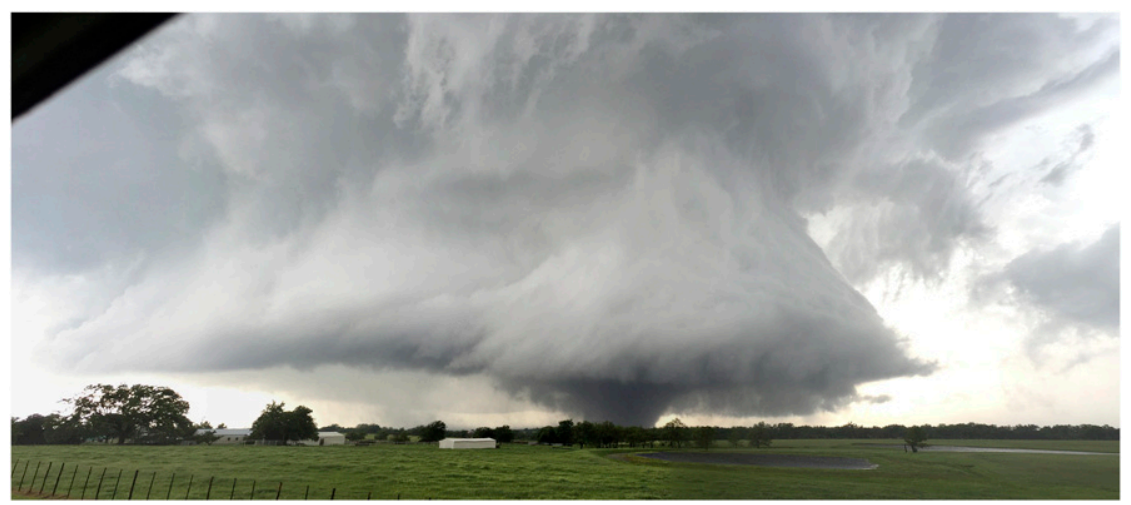

FIG. 11. A wide-angle, panoramic photograph of the updraft and tornado to the north of Sulphur and to the west-southwest of RaXPol near 2155 UTC. (Photo courtesy of D. Reif.)

(Fig. 13). Along the east side of this feature at these higher elevation angles was an anticyclonic couplet, with a cyclonic couplet evident on the western side of this band. This anomaly was located from $\sim 6$ to $\sim 11 \mathrm{~km}$ to the north-northeast of the radar; the greater distance between RaXPol and this feature (compared to RaXPol's range from the first anomaly $\sim 10-15 \mathrm{~min}$ earlier), combined with the favorably unlevel radar truck, yielded beam heights sufficiently high to sample this feature.

The general structure of the $V_{R}$ field seen by RaXPol near 2200 UTC is quite similar to that seen by the WSR$88 \mathrm{D}$ radars approximately $12 \mathrm{~min}$ earlier (Fig. 14). The reader is reminded that RaXPol, which was south of the supercell, had a different viewing angle than the WSR88Ds, which were north-northwest of the supercell. As such, the sign of the $V_{R}$ data from RaXPol is nearly the opposite of that from the WSR-88D radars. Regardless, we see the same general structure in these two features-a west-east band of enhanced southerly flow flanked by cyclonic shear on the west side and anticyclonic shear on the east side. In the next section, we will show that neither of these couplets was likely associated with a tornado.

\section{Discussion}

Because KTLX was operating in Multiple Elevation Scan Option for Supplemental Adaptive IntraVolume Low-level Scan (MESO-SAILS; Chrisman 2014a,b) mode on this day, $0.5^{\circ}$ scans were collected every $\sim 70$ $110 \mathrm{~s}$. This was beneficial for tracking the main tornado, but it resulted in lengthy update/revisit times (i.e., $\sim 6.5 \mathrm{~min}$ ) on elevation angles above $0.5^{\circ}$. The comparatively infrequent sampling above the lowest elevation angle made it difficult to track and examine the evolution and vertical structure of these features. KOUN, since it was operating in a sector scan mode, was able to scan all elevation angles every $\sim 90-95 \mathrm{~s}$, which substantially increases the ease with which these features can be tracked and lets us examine the vertical structure of these features with much greater temporal granularity. In this particular case, the anticyclonic couplet associated with the first anomaly just happened to be most intense on the $0.5^{\circ}$ scans when KTLX was otherwise scanning through the middle and upper elevation angles of its VCP around 2147-2149 UTC, which means that we do not have data from KTLX on the scans immediately above the $0.5^{\circ}$ scan (i.e., $0.9^{\circ}$ and $1.3^{\circ}$ ) during this time. The faster update times provided by KOUN provide very important information not available from KTLX.

To elucidate the vertical structure of the anticyclonic couplet, data from KOUN's lowest three elevation angles during this time are shown in Fig. 15. The center of the anticyclonic couplet shifted eastward with height; the couplet at $0.9^{\circ}$ (yellow circle) and the anticyclonic shear area at $1.4^{\circ}$ (white circle) were $\sim 3$ and $\sim 5 \mathrm{~km}$, respectively, to the east of the couplet on the $0.5^{\circ}$ elevation angle scan (black circle). Because only $12 \mathrm{~s}$ elapsed between each panel/elevation angle and because the general movement of the feature northward was nearly perpendicular to the horizontal shift of the couplet (i.e., eastward with height), the artificial tilt produced by the movement of the feature between scans was small compared to the actual displacement of the couplet with height. The tilt of the couplet with height is perhaps better seen in a reconstructed vertical cross-section taken across the couplet (Fig. 16). At $\sim 2147$ UTC, the center of the couplet on the lowest elevation angle scan $\left(0.5^{\circ}\right)$ was $\sim 3 \mathrm{~km}$ to the west of the couplet at $0.9^{\circ}$ and $\sim 4-5 \mathrm{~km}$ west of the center of the anticyclonic shear area at $1.4^{\circ}$. As noted previously, cyclonic shear was present to the west of the anticyclonic couplet, though the amount of gate-to-gate shear associated 


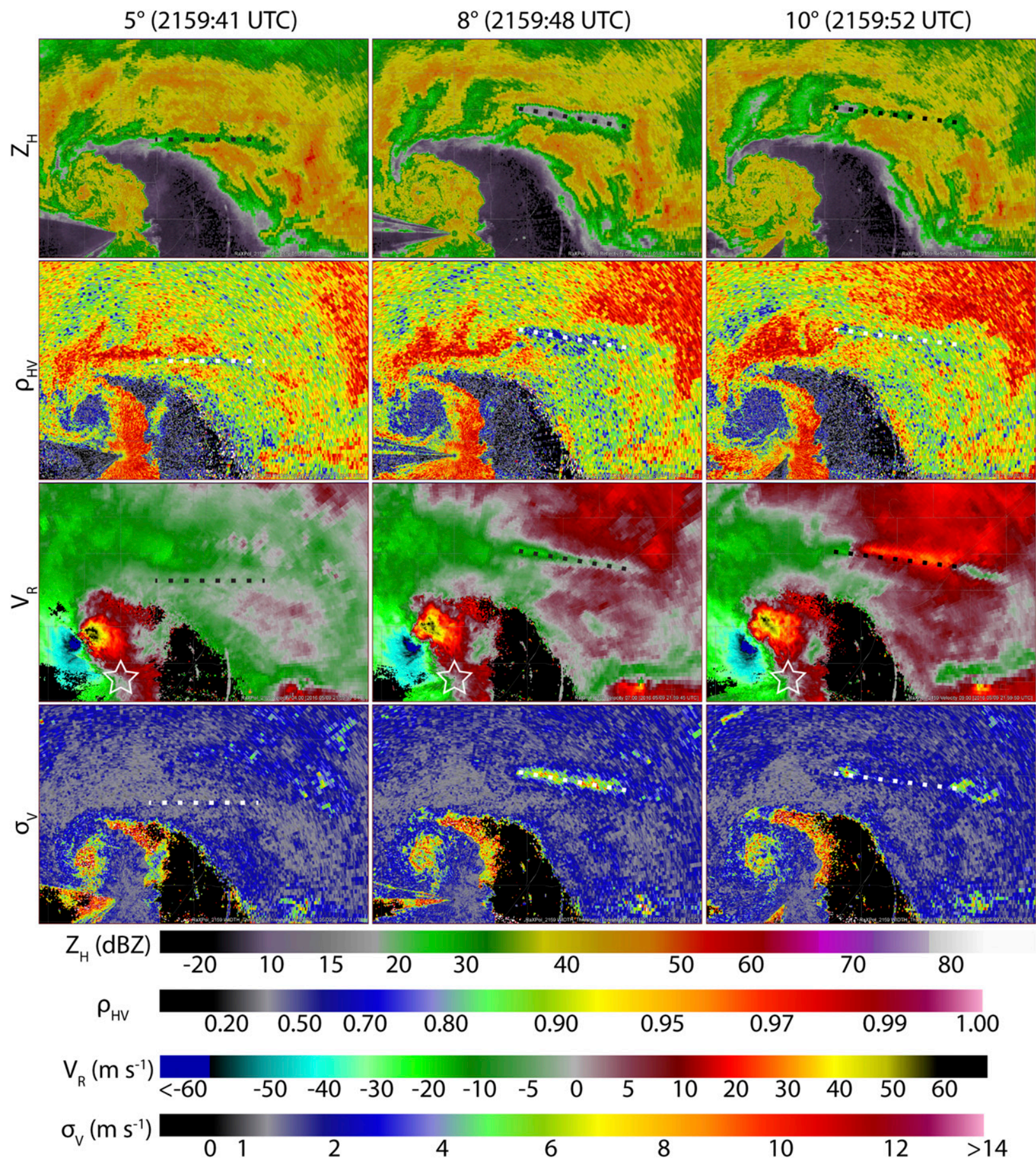

FIG. 12. RaXPol-observed (from top to bottom) $Z_{H}, \rho_{\mathrm{hv}}, V_{R}$, and $\sigma_{v}$ at (left) $5^{\circ}$, (center) $8^{\circ}$, and (right) $10^{\circ}$ elevation angles shortly before 2200 UTC. The dotted black and white lines mark the location of a narrow band of reduced $Z_{H}$. RaXPol's location is marked by the white star in the $V_{R}$ images.

with this cyclonic couplet was generally less than that associated with the anticyclonic couplet on the lowest scan.

The structure of the $V_{R}$ field-the elongated enhancements in $V_{R}$ and "stretched out" nature of the velocity couplet-is similar to another case that RaXPol observed several years prior. On the evening of 25 May 2012, RaXPol scanned a nearly horizontally oriented tornado as it "roped out" near Russell, Kansas (Fig. 17a). The extreme tilt of that tornado as it was dissipating resulted in a very elongated structure to the enhanced 


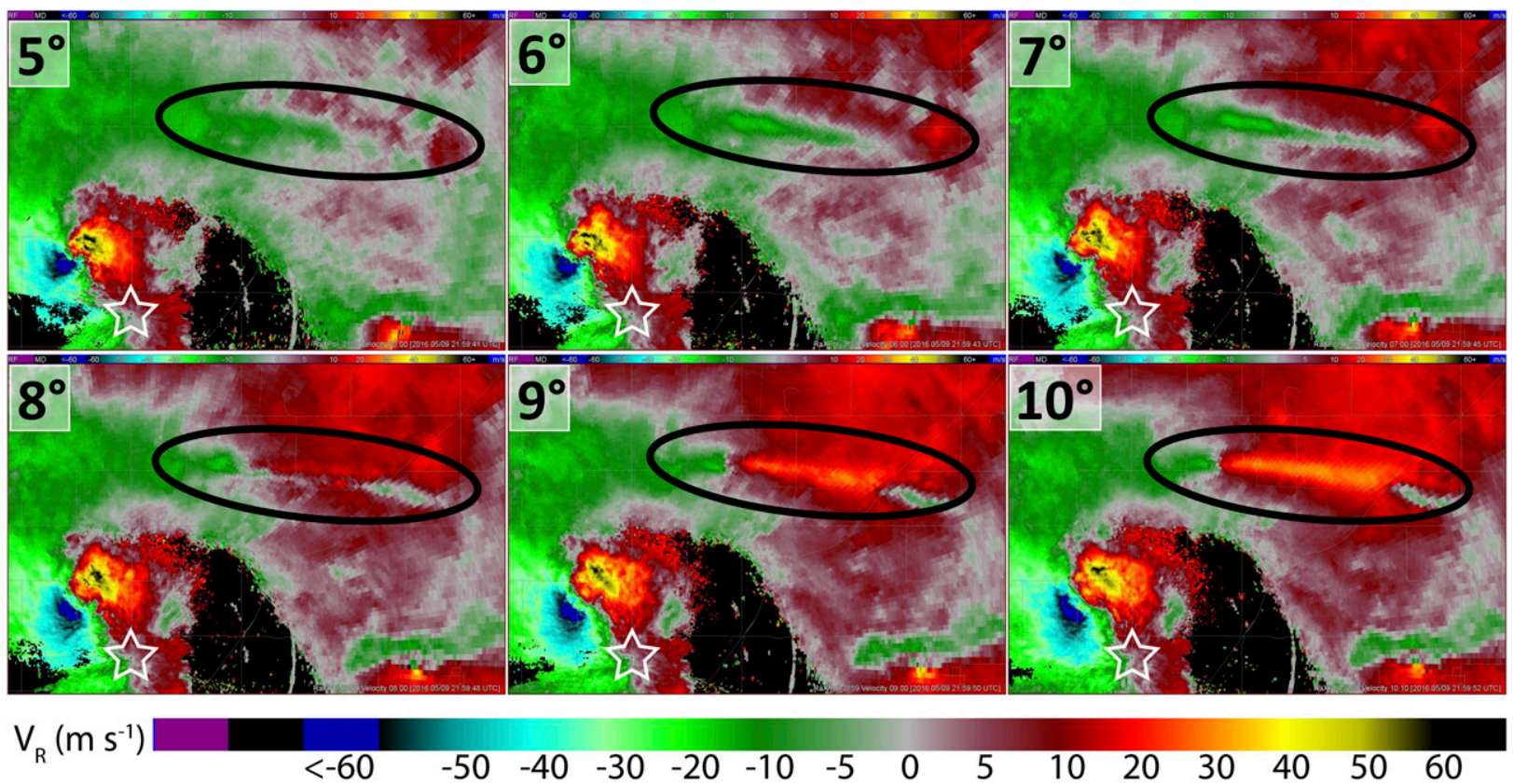

FIG. 13. The vertical structure of the second quasi-linear $V_{R}$ anomaly shortly before 2200 UTC as seen on $5^{\circ}-10^{\circ}$ elevation angle scans from RaXPol. RaXPol's location is marked by the white star.

velocity couplet as the inbound and outbound extremes occupied a relatively large azimuthal sector (Fig. 17b; Bluestein 2020, Fig. 24). This general structure looks quite similar to the elongated velocity anomalies and couplets observed on 9 May 2016.

At the range of these features from the two WSR-88D radars (i.e., $\sim 80-90 \mathrm{~km}$ from KTLX and $\sim 80-85 \mathrm{~km}$ from KOUN), the $1.0^{\circ}$ half-power $(3 \mathrm{~dB})$ beamwidth was $\sim 1.2 \mathrm{~km}$. Consequently, not only was the main lobe of the radar beam on the $0.5^{\circ}$ elevation angle scan centered near $1.4 \mathrm{~km}$ above radar level (ARL), ${ }^{4}$ the beam (as defined by the bounds of the 3-dB beamwidth) actually extended from $\sim 0.9$ to $\sim 2.1 \mathrm{~km} \mathrm{ARL}^{5}$ - see the white lines in Fig. 16. Similarly, though centered near $2.0 \mathrm{~km}$ ARL, the main lobe of the second lowest elevation angle scan $\left(0.9^{\circ}\right)$ extended from $\sim 1.5$ to $2.7 \mathrm{~km}$ ARL. The relatively coarse vertical resolution, even though the lowest elevation angles are oversampled,

\footnotetext{
${ }^{4}$ KTLX and KOUN are located $\sim 390$ and $377 \mathrm{~m}$ above sea level (MSL), respectively. RaXPol was located $\sim 354$ and $\sim 330 \mathrm{~m}$ MSL at the first and second deployment locations. For the most part, ARL heights from KTLX and KOUN will be $\sim 20-50 \mathrm{~m}$ higher than the ARL heights from RaXPol.

${ }^{5}$ These beam height estimates assume normal propagation characteristics. The actual beam propagation probably deviated from the assumed model (i.e., a standard refractivity vertical profile) because of the likely presence of stronger low-level static stability.
}

limited the amount of detail of the vertical structure of the anticyclonic couplet that the radar could provide. In addition, no part of the main beam (within the $3 \mathrm{~dB}$ bounds) was below $\sim 0.9 \mathrm{~km}$ ARL. The lack of nearground data significantly increases the difficulty of assessing whether a particular velocity couplet may be associated with a tornado (or with the parent tornado cyclone/anticyclone or mesocyclone/mesoanticylone) because tornadic and nontornadic mesocyclones (or mesoanticyclones) may look strikingly similar even a few hundred meters above ground (e.g., Trapp 1999).

In contrast to the limited low-level data availability and resolution provided by the WSR-88D radars, the close proximity of RaXPol to the supercell resulted in more complete data coverage and much better resolution in the lower troposphere. Whereas KOUN and KTLX provided no data coverage below $\sim 0.9 \mathrm{~km}$ ARL (the "bottom" of the radar beam on the lowest scan), RaXPol was able to collect data at 7-9 (depending upon specific location), nonoverlapping ${ }^{6}$ or nonoversampled elevation angles below this height. Vertical crosssections through the second velocity anomaly as observed by RaXPol (Fig. 18) provide much more clarity

\footnotetext{
${ }^{6}$ The radiation pattern from the antenna is essentially continuous, so electromagnetic energy does propagate to areas well outside the radar bore sight. However, the $3-\mathrm{dB}$ beamwidth is traditionally used to define the "radar beam," and we continue that use here while acknowledging that side lobes exist.
} 


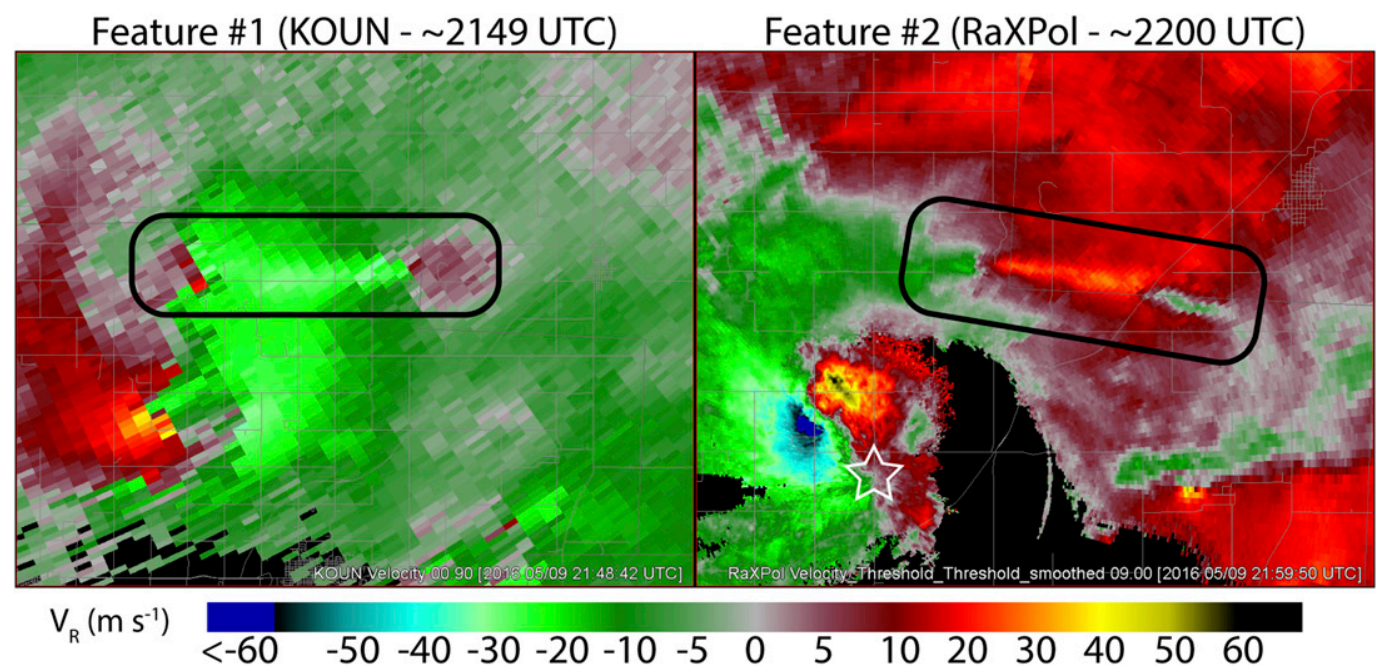

FIG. 14. Two similar-looking features (black, rounded rectangles): (left) $0.9^{\circ}$ elevation angle data from KOUN at 2148:42 UTC and (right) 9.0 elevation angle data from RaXPol at 2159:50 UTC. RaXPol's location is marked by the white star (KOUN's location is far outside the extent of the image).

on the vertical structure of the flow near this feature. The vertical cross-sections through the elongated $V_{R}$ feature appear to show a quasi-horizontal vortex centered on the elevation angle where the zonally oriented band is least apparent (i.e., $8^{\circ}$ ), and the center of this vortex appears to slope upward with eastward extent with some indication that the far western edge slopes slightly upward as well; the vortex is centered approximately 1.1-1.2 km ARL (after accounting for the pitch and roll of the radar truck), $\sim 1.0-1.1 \mathrm{~km}$ ARL, and $\sim 1.5-1.6 \mathrm{~km}$ ARL from west to east in the three crosssections provided in Fig. 18. These heights are near the height of the lowest data available from the WSR$88 \mathrm{D}$ radars.
Owing to the lack of a tornadic vortex observed by RaXPol, we hypothesize that the anticyclonic vortex that one may infer from the lowest-elevation WSR-88D data (and higher elevation angles from RaXPol) and that originally was thought to have been associated with an anticyclonic tornado that produced damage northeast of Sulphur was not a tornado after all but, rather, was a quasi-horizontal vortex. To briefly explain why this is our hypothesis, we first consider a purely horizontal vortex. Let us place a radar (such as KTLX or KOUN) to the north-northwest of such a vortex in a similar orientation as KTLX and KOUN were on 9 May 2016. If the vorticity vector were to point to the right when the radar is viewing to the south-southeast

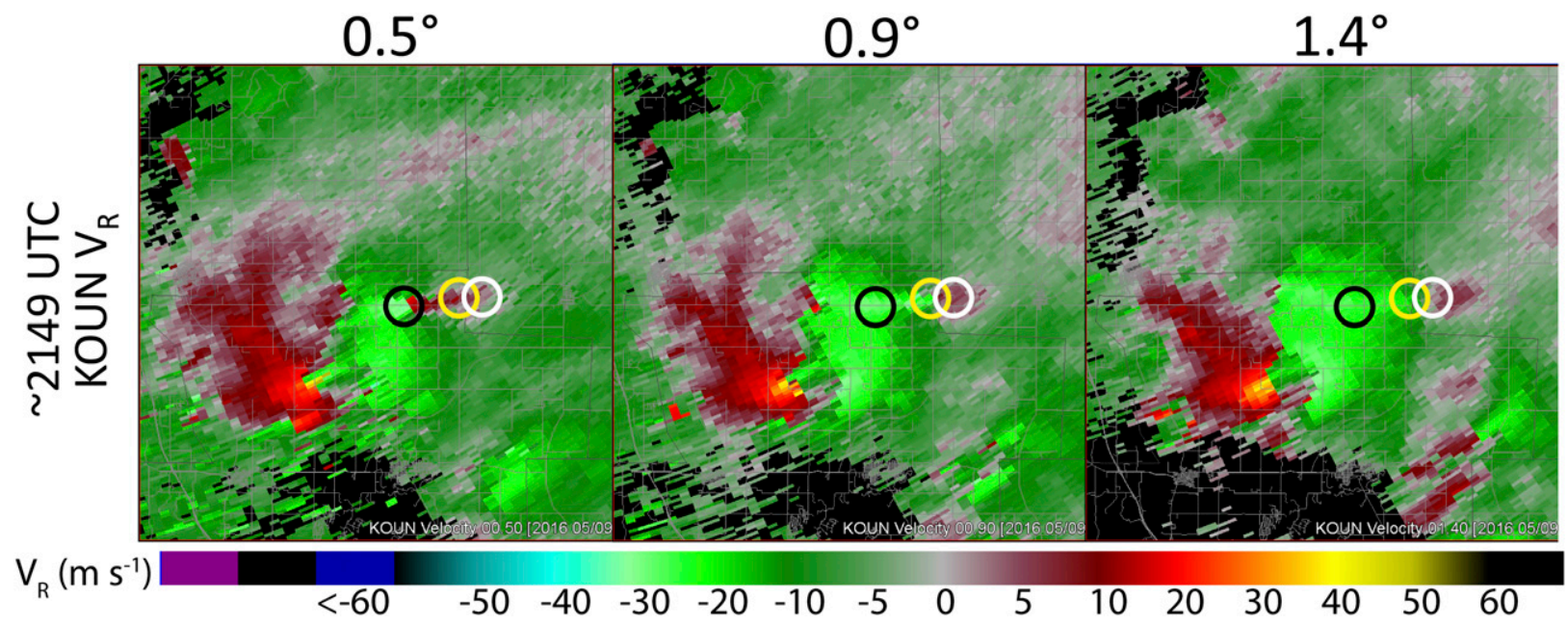

FIG. 15. $V_{R}$ at (left) $0.5^{\circ}$, (center) $0.9^{\circ}$, and (right) $1.4^{\circ}$ elevation angles from KOUN at approximately 2149 UTC, with the approximate location of the anticyclonic couplet marked by the black, yellow, and white circles, respectively. 


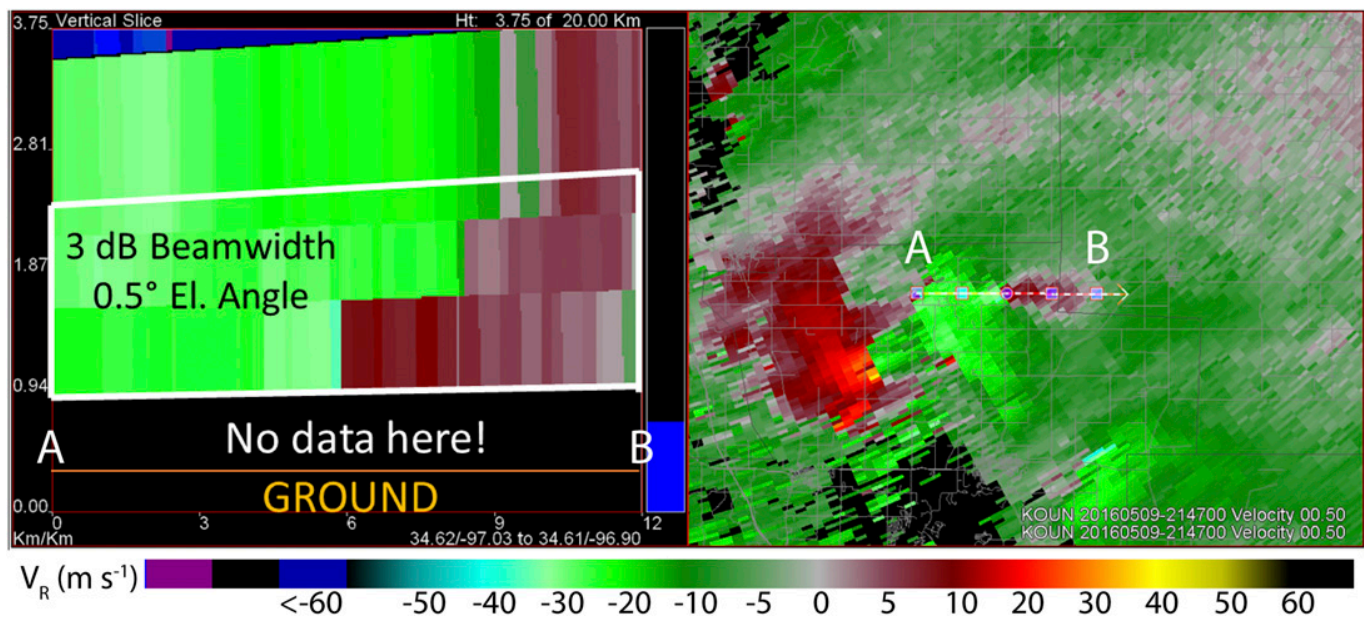

FIG. 16. (left) A west-east reconstructed vertical cross section along the line shown in the (right) $0.5^{\circ}$ elevation angle $V_{R}$ scan from KOUN at 2147 UTC. The white quadrilateral in the cross-section marks the approximate vertical "edges" (at least the 3-dB/half-power edges) of the radar beam at the lowest elevation angle. The horizontal brown line in the vertical cross section marks the approximate ground level. Heights on the ordinate of the vertical cross-section are in units of kilometers MSL.

(i.e., vorticity vector is pointing to the west-southwest), the radar would measure inbound $V_{R}$ on the top of the vortex and outbound $V_{R}$ on the bottom of the vortex (Figs. 19a,b). Now, rather than a purely horizontal vortex, consider a slightly tilted (quasi-) horizontal vortex that slopes slightly downward with westward extent (upward with eastward extent), and let us view that as we did in Fig. 16 (such that west is on the left and east is on the right; Figs. 19c,d). The tilted vortex now is associated with couplets at different elevation angles, and a vortex tilted in this manner would manifest as anticyclonic couplets that tilt eastward with height. This is strikingly similar to what KOUN observed with the first anticyclonic couplet shown in Fig. 15.
But what of the cyclonic-anticyclonic pair that was also evident in RaXPol data near 2200 UTC and for which hints may be evident in the coarser-resolution WSR-88D data? We can recreate such a $V_{R}$ field by advancing the "tilted quasi-horizontal vortex" model one step further and consider an arched quasi-horizontal vortex (Fig. 20; Bluestein 2005, Fig. 5). If a radar is placed as RaXPol was on 9 May 2016 (i.e., to the south, looking northward), a quasi-horizontal vortex that tilts downward then upward with westward extent is seen as a cyclonic-anticyclonic vortex pair for elevation angles that cut through the center of the vortex, and the distance between this pair increases at higher elevation angles. As above, a very similar structure appears in the $V_{R}$ data from RaXPol shown in Fig. 13. In those

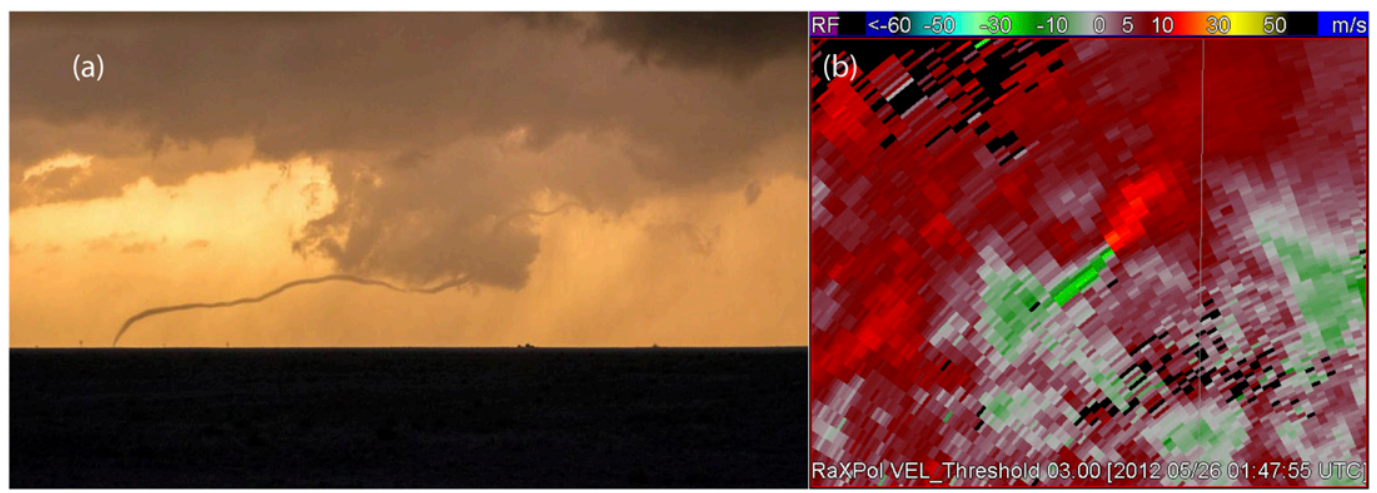

FIG. 17. (a) A photograph (courtesy of J. Snyder) of the dissipating ("rope out") stage of a tornado that occurred southwest of Russell, Kansas, on the evening of 25 May 2012. (b) Corresponding $V_{R}$ image from RaXPol at $\sim 0148$ UTC 26 May 2012 at 3.0 elevation angle. 


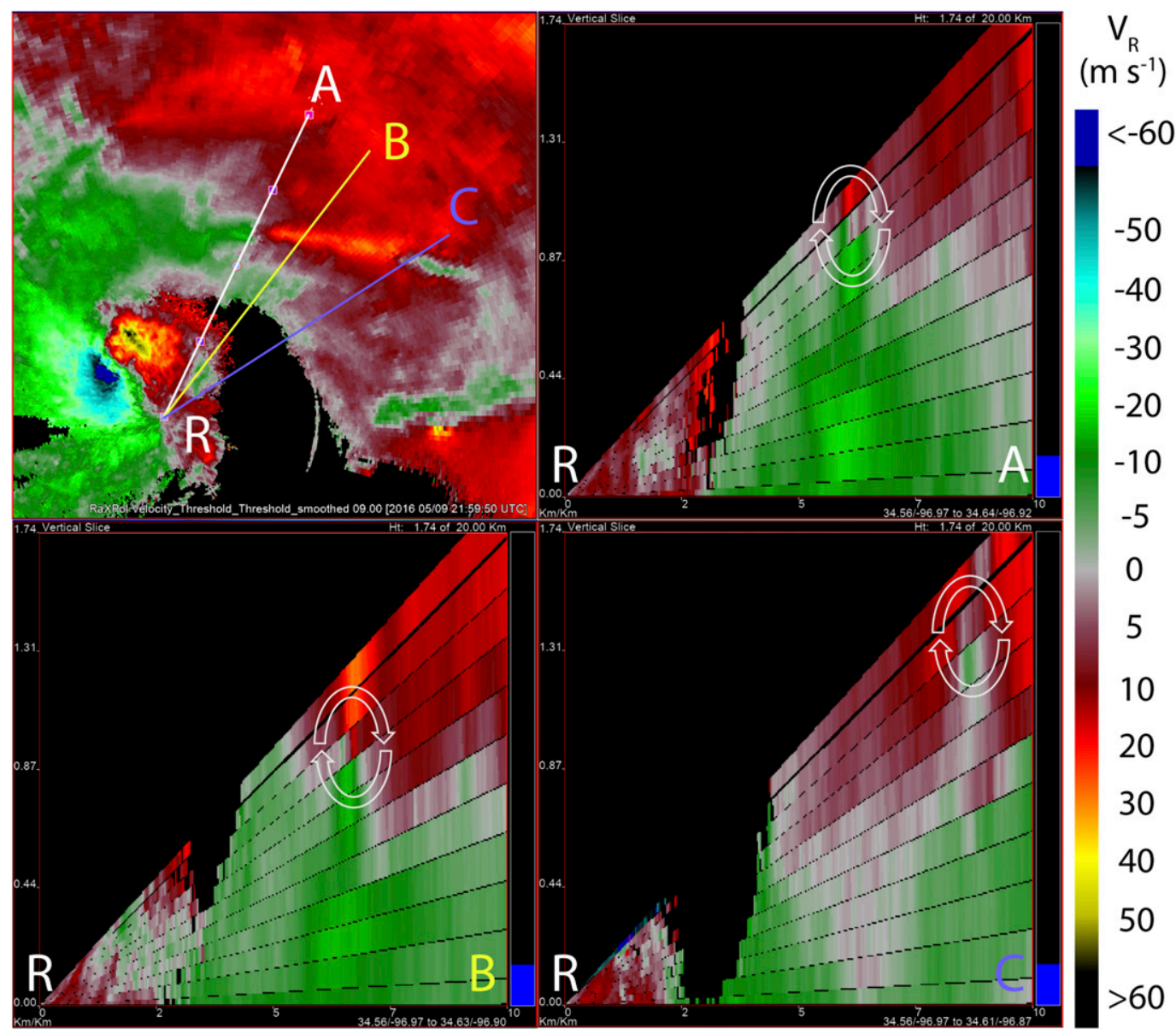

FIG. 18. Shown is $V_{R}$ from RaXPol at $9.0^{\circ}$ elevation angle and three reconstructed vertical cross sections through the quasi-horizontal $V_{R}$ vortex near 2159:48 UTC. The cross sections in the top right, bottom left, and bottom right correspond to those along the white (R-A), yellow (R-B), and purple (R-C) lines in the $9.0^{\circ}$ PPI (top left), respectively. Heights on the ordinate of the vertical cross sections are in units of kilometers ARL without adjustment for nonzero pitch and roll.

observed data, the lower elevation angles are primarily characterized by inbound (i.e., northerly or northeasterly) flow. Between $7^{\circ}$ and $8^{\circ}, V_{R}$ drops to near $0 \mathrm{~m} \mathrm{~s}^{-1}$ along the axis of the band. At the two highest elevation angles at which data were collected (i.e., $9^{\circ}$ and $10^{\circ}$ ), the strongest flow is outbound (i.e., southerly or southwesterly), and the cyclonic and anticyclonic couplets get farther apart with increasing height, just as in the schematic for an arched quasi-horizontal vortex shown in Fig. 20.

Though the authors are not aware of radar observations of quasi-horizontal vortices (be they actually horizontal, slightly tilted, or arched) in the literature similar to those seen on 9 May 2016 in the forward-flank echo of a supercell, there are observations of quasi-horizontal vortices elsewhere in supercells. For example, quasihorizontal vortices have been seen in high-resolution simulations (e.g., Orf et al. 2017; Oliveira et al. 2019) and observations from mobile radars (e.g., Wurman and Kosiba 2013; Houser et al. 2016) to the south of strong tornadoes, perhaps associated with internal rear-flank downdraft surges and/or near-ground frictional effects. However, these quasi-horizontal vortices are generally smaller in size and occur in a distinctly different part of the storm compared to the two examined in this paper. A different "type" of quasi-horizontal or arched vortex was observed in a tornadic supercell that occurred in eastern Oklahoma on 26 May 1997 far removed from the ongoing cyclonic tornado and above the supercell's cloud base (Fig. 21a; Bluestein 2005). Yet another tilted vortex was seen as a northward-pointing elevated funnel cloud in a tornadic supercell that occurred on the evening of 12 May 2004 in southern Kansas (Fig. 21b; Bluestein et al. 2007b). These latter two examples occurred north or northeast of the tornado, though neither appear to be particularly similar to those observed 
(a)

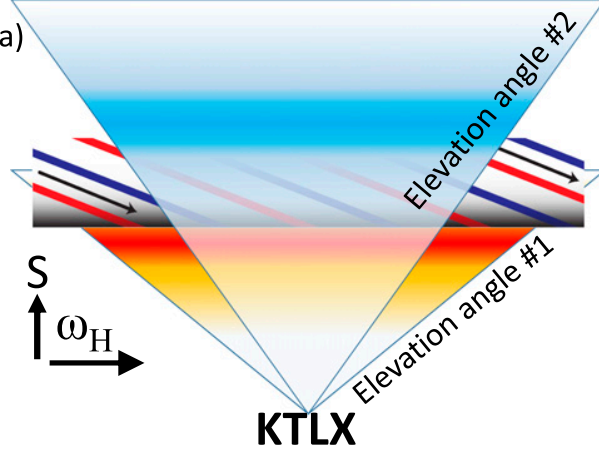

(b) Vertical cross-section through vortex

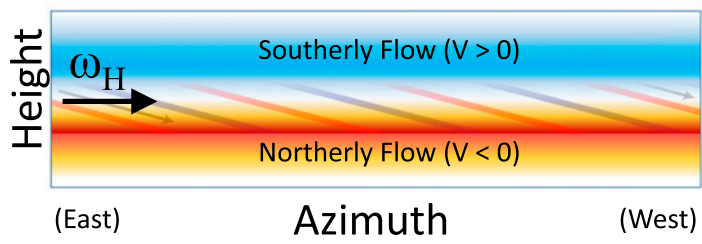

Blue is towards KTLX radar

Red is away from KTLX radar

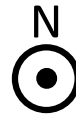

(c)

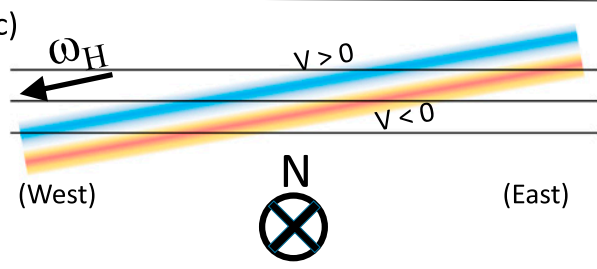

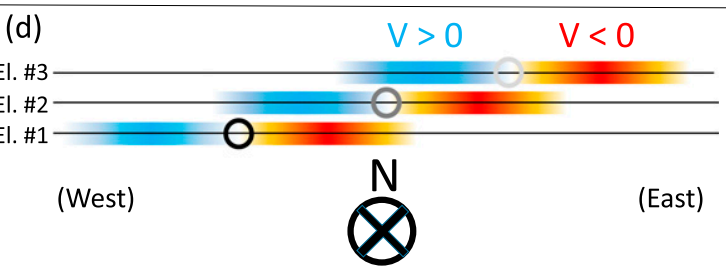

FIG. 19. (a) A horizontal vortex as scanned by a radar located to the north of the vortex, as with KTLX (looking to the south), with the vorticity vector $\omega_{H}$ directed to the right (west). (b) The horizontal vortex viewed along a crosssection through the center of the vortex. (c) A quasi-horizontal vortex, viewed from a radar located to its north, tilted upward from left (west) to right (east), with three elevation angles represented by the three horizontal lines. (d) The representation of the radial velocity of the tilted vortex at three elevation angles; black, dark gray, and light gray circles denote the approximate location of the center of the vortex at the bottom, middle, and top elevation angle, respectively. In (a) and (b), the horizontal vortex is illustrated as a "barber pole." In all panels, blue represents flow toward the radar, and red represents flow away from the radar. In this case, with the radar to the north scanning to the south, blue represents southerly flow (i.e., positive meridional component $V$ ), and red represents northerly flow $(V<0)$. This is similar to the observed data of the tilted tornado shown in Fig. 17 except for minor differences in orientation. Note that south is up in (a); north is out of the page in (b) (as in looking from the perspective of the KTLX radar southward toward the vortex with the north arrow directed into the radar), and north is into the page in (c) and (d) (so that west is on the left and east is on the right as commonly depicted in popular maps).

herein in size nor did they extend into the forward-flank echo as much as these vortices examined herein.

While this quasi-horizontal or slightly arched model fits with the observed $V_{R}$ patterns (and enhanced $\sigma_{v}$, given the likelihood of enhanced shear in radar volumes sampling within such a vortex), it does not necessarily explain the structures seen in $Z_{H}$ and $\rho_{\mathrm{hv}}$. It is possible that vertical motions associated with the quasi-horizontal vortices may have reduced scatterer concentrations and thus have been responsible for the observed reduction in $Z_{H}$. There are several possible explanations for the observed reduction in $\rho_{\mathrm{hv}}$ in the narrow region of low $Z_{H}$, though we wish we could be more confident in positing which explanation is responsible. The reduced $\rho_{\mathrm{hv}}$ may have been caused by multipath scattering occurring in the presence of hail, by sidelobe contamination, or by the presence of nonmeteorological scatterers. The relatively low SNR (not shown but can be inferred from the low $Z_{H}$ ) may have contributed to a noise bias. Unfortunately, differential attenuation complicated analysis of $Z_{\mathrm{DR}}$, and the very narrow nature of the band reduces the utility of specific differential phase $K_{\mathrm{DP}}$ since $K_{\mathrm{DP}}$ is comparatively low resolution owing to the way in which it is calculated. Overall, the polarimetric fields do not provide a lot of evidence one way or another.

The origin of the quasi-horizontal vorticity associated with these features is unknown, though we can present three possible explanations. First, the quasi-horizontal vortices may have been manifestations or remnants of the horizontal convective rolls seen on the visible satellite imagery that clearly showed cloud streets during the afternoon in the marginally cooler air to the north of the outflow boundary (Fig. 2b). The 1800 UTC HRRR forecast sounding valid near the Katy-Sulphur supercell valid $\sim 2100$ UTC (Fig. 2c) indicates a stable layer $\sim 1-$ $1.5 \mathrm{~km} \mathrm{AGL}$, which is close to the height at which RaXPol appeared to show the center of the quasihorizontal vortices, though the thermal profile in that forecasting sounding is unable to be verified. It is possible that the quasi-horizontal vortices were deflected downward as a result of downbursts within the forward-flank downdraft. It is difficult to say with great confidence and 
(a)

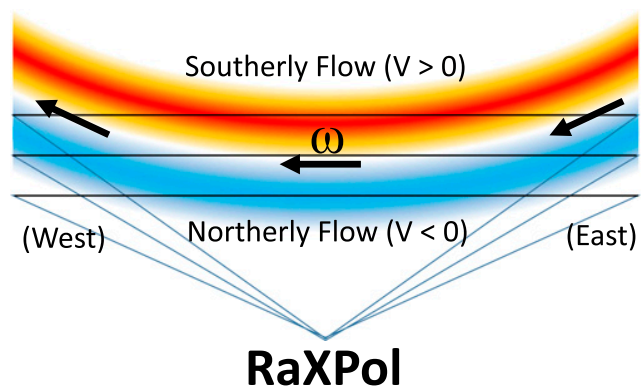

(b)

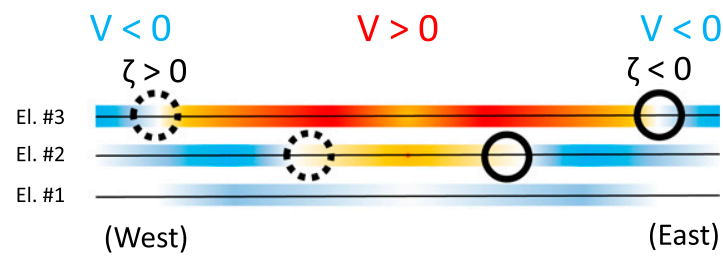

Blue is towards RaXPol

Red is away from RaXPol

FIG. 20. (a) An illustration of a vertical cross section of $V_{R}$ along an arched quasi-horizontal vortex as viewed from the south looking to the north, as with RaXPol's position on 9 May 2016. View is such that west is to the left and east is to the right, and the north arrow points into the page. Black arrows are vorticity vectors. (b) The $V_{R}$ cross section at three elevation angles indicating centers of cyclonic (dashed black circles) and anticyclonic (solid black circles) shear. As in Fig. 19, $V$ represents the meridional component of the flow. Note that the viewing angle of RaXPol in this figure is nearly the opposite of that of KTLX in Fig. 19, so the sign of $V_{R}$ (and thus the colors) in this figure is essentially reversed from that in Fig. 19.

using only the data available why such vortices would have intensified enough to produce the observed, prominent $V_{R}$ features. However, these quasi-horizontal rolls or vortices may have been quasi-horizontally stretched as flow through the southern periphery of the forward-flank echo accelerated westward and southwestward into the updraft or low-level mesocyclone associated with the intense, cyclonic tornado. As shown by Wade et al. (2018), an examination of soundings collected within the near and far fields of tornadic and nontornadic supercells has revealed that the tornadic supercells may enhance the near-storm low-level flow compared to the flow farther from the supercell. While the group that collected the soundings reported in Wade et al. (2018) did collect several soundings on 9 May 2016, they did not collect a near-storm sounding during the time of the Sulphur tornado (M. Coniglio 2016, personal communication).

Second, the quasi-horizontal vortices may have been associated with boundaries or processes internal to the supercell, such as with a rotor above/along the southern edge of downbursts (e.g., Droegemeier and Wilhelmson 1986; Kessinger et al. 1988) within the forward-flank
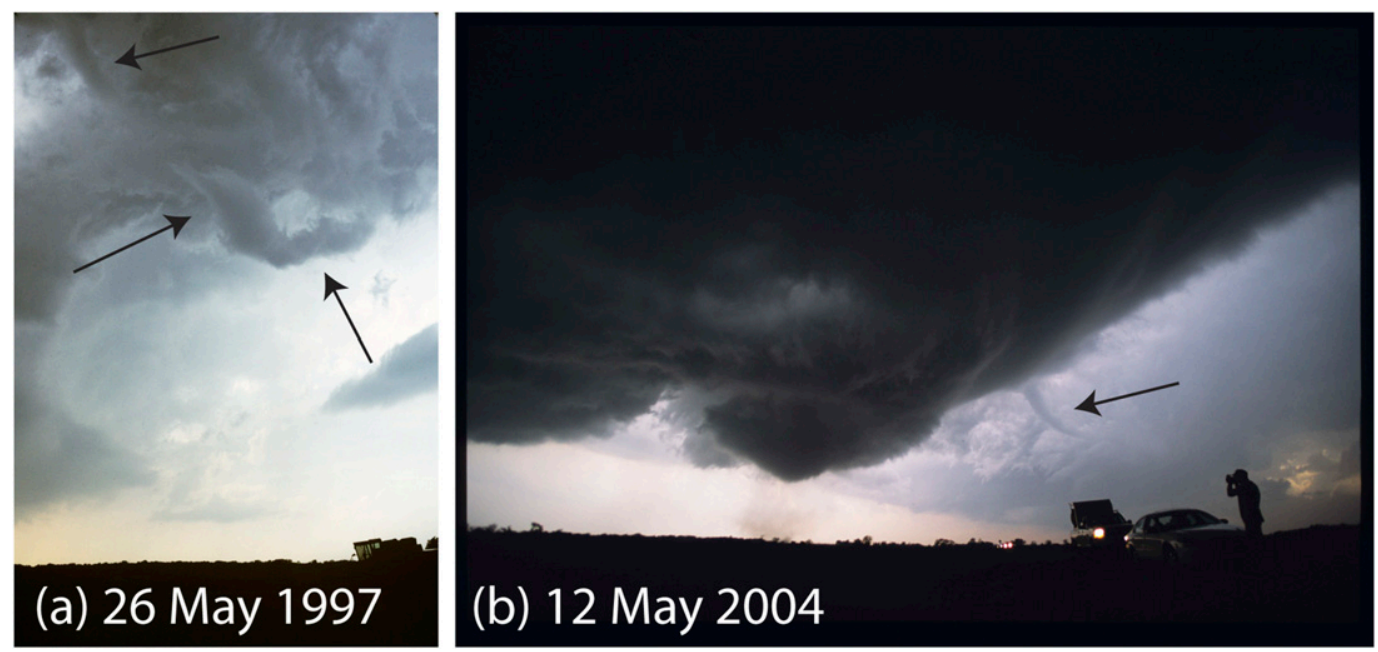

FIG. 21. (a) A photograph of a funnel cloud associated with an arched vortex aloft that is "attached" to the cloud associated with the updraft (far left side) of a tornadic supercell on 26 May 1997 in eastern Oklahoma. (b) A wideangle photograph, taken looking to the west, of a tornadic supercell with a developing tornado (just left of center) near Attica, Kansas, on the evening of 12 May 2004. The black arrow in (b) highlights an apparent elevated funnel cloud that appears to point to the north. The photograph in (b) appears in Fig. 4 in Bluestein et al. (2007b). (Photographs courtesy of H. Bluestein.) 
region of the supercell or with a baroclinic zone of some sort [perhaps something similar to the left-flank convergence boundary described by Beck and Weiss (2013)]. During the first $V_{R}$ anomaly between $\sim 2144$ and 2152 UTC, RaXPol observed the development of a downburst to the north of the location of the quasihorizontal vortex, with the strongest near-ground inbound $V_{R}$ (i.e., flow with a northerly or northeastern component) below the quasi-horizontal vortex. Unfortunately, we do not have sufficient near-ground, in situ thermodynamic observations to identify and quantify in-storm baroclinic boundaries.

A third possibility is that the quasi-horizontal vortices may have been generated by convective storms that developed to the immediate southeast of the KatySulphur (A) supercell and merged into the forwardflank part of the storm or by the interaction between the gust fronts associated with them. There were several instances in which northward-moving precipitation merged into the southern edge of the forward flank echo, resulting in a narrow band of comparatively low $Z_{H}$ between the two areas of precipitation. The cold pools associated with the merging convection likely did not directly produce the observed quasi-horizontal vortices since the baroclinically generated vorticity would have been oriented in the opposite direction (e.g., eastward for a density gradient that points to the south as it would along the north edge of a cold pool). However, it is possible that other factors may have produced or been associated with horizontal vorticity (e.g., boundaries associated with the interaction between the primary Katy-Sulphur supercell and flow around or modulated by the merging convection). Unfortunately, we do not have any high-resolution thermodynamic and any additional kinematic data independent of the radars to identify boundaries (either at the ground or aloft) that may have accompanied the merging convection.

Regardless of which (if any) of these explanations were responsible for these signatures, we opt not to speculate further as to why quasi-horizontal vortices like those thought to have been observed on this day are not observed more often, particularly since several of the explanations describe not-uncommon conditions or scenarios. It is possible that these quasi-horizontal vortices are not actually very rare in supercells but that they were just particularly intense in this case for some asyet-unknown reason.

Though likely unrelated, RaXPol observed multiple low-reflectivity ribbons (LRRs; Wurman et al. 2012; Kosiba et al. 2013; Snyder et al. 2013) in the KatySulphur supercell (e.g., left side of Fig. 12). LRRs examined by Griffin et al. (2018) were often associated with cyclonic vertical vorticity, tended to move rearward with time, and were often associated with upward motion below $\sim 2 \mathrm{~km}$ AGL, at least until they began to dissipate. Although the two quasi-horizontal vortices examined herein were associated with relatively low RaXPol-observed $Z_{H}$ at times, their location was not really similar to the LRRs that have been described in the literature so far. LRRs typically occur at the very rear of the forward flank echo where the hook echo "begins" and are usually oriented in a similar direction as the forward flank echo $Z_{H}$ gradient (i.e., described LRRs tend to be oriented in a general north-south manner), which is not really how the bands of reduced $Z_{H}$ associated with these quasi-horizontal vortices were oriented. However, as Griffin et al. (2018) noted, nascent LRRs can develop along the trailing (i.e., western for supercells oriented like they were on 9 May 2016) edge of the forward flank $Z_{H}$ gradient to the northeast of the hook echo, which is more similar to what we see in the quasi-horizontal vortices here. Regardless, at this time, we do not consider the two vortices here to be LRRs. Despite the LRRs observed by RaXPol to the west of the quasi-horizontal vortices in the supercell examined herein, it is not immediately apparent how (if at all) they are connected to the two main $V_{R}$ anomalies and suspected quasi-horizontal vortices that serve as the focus of this paper, though it is not possible to say with certainty that they are completely unrelated using only the radar information available for this case.

\section{Summary}

Several tornadic supercells moved across Oklahoma on the afternoon of 9 May 2016, one of which, on the lowest scan available from nearby WSR-88Ds, contained a strong anticyclonic vortex signature that moved northward into the forward-flank region of a tornadic supercell. The presence of a swath of damage near the vortex led to the preliminary determination that this peculiarly placed vortex was an anticyclonic tornado. The high-resolution data provided by RaXPol, at least during the time of data collection, do not show that an anticyclonic tornado moved northward into the forward flank echo region as may be inferred by the WSR-88D data. Although the WSR-88D data could be interpreted to show that an anticyclonic vortex of some sort occurred, we hypothesize that the features that were sampled were instead quasi-horizontal or arched quasihorizontal vortices centered $\sim 1.0-1.5 \mathrm{~km}$ AGL. The simple illustrations for what such a feature would look like in $V_{R}$ data (i.e., Figs. 19,20) are very similar to the structure of the $V_{R}$ fields from both the WSR-88D radars and RaXPol. 
While it does not appear that the anticyclonic couplets were associated with a peculiarly located anticyclonic tornado as initially thought, we do not have sufficient evidence to speculate as to the source of the damage. As noted previously, no damage was reported or relayed to the OUN NWSFO during or after the event, which is rather peculiar considering the photographs taken by the NWS survey team include EF1 damage to singlefamily houses. It is possible that the damage came from another storm at a different time-for example, on the morning of 9 May 2016, storms moved through the area, and very weak rotation is seen on at least one scan from KTLX within the early day storms, though, again, damage was also not reported to the NWS at this earlier time. It is also possible that the winds that produced the damage were associated with a downburst such as that observed by RaXPol (Fig. 9), though there was not a strong signal for divergence in the damage, and the lengthy-but-narrow geometry of the damage swath is more reminiscent of a tornado track than of a downburstrelated damage swath. Of course, it remains possible that a tornado with a $\sim 15-20-\mathrm{km}$ track did occur in this area but was not seen by RaXPol, though this seems extremely unlikely, at least during the period during which the anticyclonic couplets were observed by the WSR-88Ds.

The limitations of the existing radar network complicate the analysis of the couplets observed by the operational KTLX radar, as spatiotemporal resolution was largely insufficient to discern the nature of the observed anticyclonic vortex couplets. The faster volume update times provided by KOUN and RaXPol allowed us to better understand how the couplets evolved with time, which was even more important in this case because of the extended revisit period above the lowest $\left(0.5^{\circ}\right)$ scan from KTLX associated with the use of MESO-SAILS. In addition, the much better spatial resolution provided by the much closer proximity of RaXPol to the supercell of interest provided the best evidence that the couplets were not associated with anticyclonic supercells; RaXPol's closer proximity to the supercell allowed it to sample much nearer to the ground and with much higher resolution than KTLX and KOUN could. Of course, the observation that high-resolution and/or low-altitude radar data may improve the detection of tornadoes is not novel. However, many previous studies that examined the advantages of close-range, high-resolution radar observations did so by highlighting when such data can detect "features"- such as tornadoes-that are otherwise not resolvable in lower-resolution, farther-aboveground data. In this paper, by contrast, we approached this from the other direction by examining when nearground, high-resolution radar data can be used to show that a tornado likely did not occur, despite what may be inferred in lower-resolution, higher minimum altitude data. While it has been shown that more closely spaced radar networks should improve tornado warning performance and potentially reduce tornado casualties as a result (e.g., Cho and Kurdzo 2019), this case provides an example of a potentially lesser known benefit that a more closely spaced radar network (e.g., McLaughlin et al. 2009) may provide.

While the additional lowest-level scans can provide critical information about the location and intensity of low-level rotation, the use of MESO-SAILS increases volume update times. As a result, assessing the evolution of convective storms, an assessment that benefits from being able to examine data aloft, can become more difficult, with modified or potentially degraded performance of algorithms [e.g., $Z_{\mathrm{DR}}$ columns (Snyder et al. 2015; Kuster et al. 2019), VIL, or MESH (Reinhart and Kingfield 2018)] that rely upon volumetric information. Of course, when a tornado is occurring or suspected, it may be of greatest importance to scan the lowest level as often as possible to provide the best estimate of the tornado's location and evolution. The eventual arrival and deployment of faster scanning radars-including those based on phased array technology - should allow meteorologists to better assess storm intensity, structure, and evolution (e.g., Heinselman et al. 2008; Kuster et al. 2015, 2016; Wilson et al. 2017a,b) above the lowest level by providing faster volumetric updates.

Acknowledgments. Funding for CK was provided by NOAA/Office of Oceanic and Atmospheric Research under NOAA-University of Oklahoma Cooperative Agreement NA11OAR4320072, U.S. Department of Commerce. RaXPol is supported by NSF Grants AGS1947146, AGS-1560945, and AGS-1262048 and by NSF MRI Grant AGS-0934307 awarded to HBB and the University of Oklahoma. RaXPol is currently adeptly maintained by Advanced Radar Research Center (ARRC) engineers and technicians. We appreciate Gabriel Garfield and Robert Prentice for providing details on and discussions about the damage survey. We thank Patrick Skinner for constructive comments and suggestions that improved this manuscript.

Data availability statement: The VIIRS imagery ("Imagery Resolution 6-Min L1 Swath SDR 375m") was acquired from the Level-1 and Atmosphere Archive and Distribution System (LAADS) Distributed Active Archive Center (DAAC), located in the Goddard Space Flight Center in Greenbelt, Maryland (https:// ladsweb.nascom.nasa.gov/), and is part of the NPP VIIRS collection 1.0, ArchiveSet 5000; more information 
is available at https://ladsweb.modaps.eosdis.nasa.gov/ missions-and-measurements/products/NPP_VIAES_L1. Oklahoma Mesonet data are accessible on http:// www.mesonet.org/index.php (doi:10.15763/dbs.mesonet). KTLX radar data were obtained from the NOAA Big Data Project hosted by Amazon Web Services. RaXPol and KOUN data are stored locally and can be accessed upon request to the authors. The model data used in this paper were from the "hysplit.20160509.18z.hrrra" file from the HRRR.V1 accessed through the NOAA ARL Archived Meteorology page (https:/www.ready.noaa.gov/ READYamet.php).

\section{REFERENCES}

Atlas, D., 1963: Radar analysis of severe storms. Severe Local Storms, Meteor. Monogr., No. 27, Amer. Meteor. Soc., 177-220.

Beck, J., and C. Weiss, 2013: An assessment of low-level baroclinity and vorticity within a simulated supercell. Mon. Wea. Rev., 141, 649-669, https://doi.org/10.1175/MWR-D11-00115.1.

Biggerstaff, M. I., and Coauthors, 2005: The Shared Mobile Atmospheric Research and Teaching Radar: A collaboration to enhance research and teaching. Bull. Amer. Meteor. Soc., 86, 1263-1274, https://doi.org/10.1175/BAMS-86-9-1263.

Bluestein, H. B., 2005: More observations of small funnel clouds and other tubular clouds. Mon. Wea. Rev., 133, 3714-3720, https://doi.org/10.1175/MWR3080.1.

,- 2020 : Observations of tornadoes and their parent supercells using ground-based, mobile Doppler radars. Remote Sensing and Natural Hazards, K. Zhang, Y. Hong, and A. AghaKouchak, Eds., John Wiley \& Sons, Inc., in press.

— nadoes, funnel clouds, and wall clouds with a portable Doppler radar. Bull. Amer. Meteor. Soc., 70, 1514-1525, https://doi.org/ 10.1175/1520-0477(1989)070<1514:OOTWFI $>2.0 . C O ; 2$.

— other convective phenomena with a mobile, 3-mm wavelength, Doppler radar: The spring 1999 field experiment. Bull. Amer. Meteor. Soc., 81, 2939-2951, https://doi.org/10.1175/ 1520-0477(2000)081<2939:OOTAOC > 2.3.CO;2.

—_, and J. C. Snyder, 2015: An observational study of the effects of dry air produced in dissipating convective storms on the predictability of severe weather. Wea. Forecasting, 30, 79-114, https://doi.org/10.1175/WAF-D-14-00065.1.

— , A. L. Pazmany, J. C. Galloway, and R. E. McIntosh, 1995: Studies of the substructure of severe convective storms using a mobile 3-mm-wavelength Doppler radar. Bull. Amer. Meteor. Soc., 76, 2155-2169, https://doi.org/10.1175/1520-0477(1995) 076<2155:SOTSOS $>2.0 . \mathrm{CO} ; 2$.

—, M. M. French, R. L. Tanamachi, S. Frasier, K. Hardwick, F. Junyent, and A. L. Pazmany, 2007a: Close-range observations of tornadoes in supercells made with a dual-polarization, X-band, mobile Doppler radar. Mon. Wea. Rev., 135, 1522 1543, https://doi.org/10.1175/MWR3349.1.

— C. C. Weiss, M. M. French, E. M. Holthaus, R. L. Tanamachi, S. Frasier, and A. L. Pazmany, 2007b: The structure of tornadoes near Attica, Kansas, on 12 May 2004: High-resolution, mobile, Doppler radar observations. Mon. Wea. Rev., 135, 475-506, https://doi.org/10.1175/MWR3295.1.
M. M. French, I. PopStefanija, R. T. Bluth, and J. B. Knorr, 2010: A mobile, phased-array Doppler radar for the study of severe convective storms: The MWR-05XP. Bull. Amer. Meteor. Soc., 91, 579-600, https://doi.org/10.1175/2009BAMS2914.1.

, J. C. Snyder, and J. B. Houser, 2015: A multiscale overview of the El Reno, Oklahoma, tornadic supercell of 31 May 2013. Wea. Forecasting, 30, 525-552, https://doi.org/10.1175/WAFD-14-00152.1.

—, M. M. French, J. C. Snyder, and J. B. Houser, 2016: Doppler radar observations of anticyclonic tornadoes in cyclonically rotating, right-moving supercells. Mon. Wea. Rev., 144, 15911616, https://doi.org/10.1175/MWR-D-15-0304.1.

K. J. Thiem, J. C. Snyder, and J. B. Houser, 2018: The multiple-vortex structure of the El Reno, Oklahoma, tornado on 31 May 2013. Mon. Wea. Rev., 146, 2483-2502, https:// doi.org/10.1175/MWR-D-18-0073.1.

Bodine, D., M. R. Kumjian, A. J. Smith, R. D. Palmer, A. V. Ryzhkov, and P. L. Heinselman, 2011: High-resolution polarimetric observations of an EF-4 tornado on 10 May 2010 from OU-PRIME. 35th Conf. on Radar Meteorology, Pittsburgh, PA, Amer. Meteor. Soc., 3B.4, https://ams.confex.com/ams/35Radar/ webprogram/Manuscript/Paper191661/tds_paper_amsradar.pdf. , R. D. Palmer, and G. Zhang, 2014: Dual-wavelength polarimetric radar analyses of tornadic debris signatures. J. Appl. Meteor. Climatol., 53, 242-261, https://doi.org/10.1175/JAMC-D-13-0189.1.

Brock, F. V., K. C. Crawford, R. L. Elliott, G. W. Cuperus, S. J. Stadler, H. L. Johnson, and M. D. Eilts, 1995: The Oklahoma Mesonet: A technical overview. J. Atmos. Oceanic Technol., 12, 5-19, https://doi.org/10.1175/1520-0426(1995)012<0005: TOMATO $>2.0 . \mathrm{CO} ; 2$.

Brown, J. M., and K. R. Knupp, 1980: The Iowa cyclonicanticyclonic tornado pair and its parent thunderstorm. Mon. Wea. Rev., 108, 1626-1646, https://doi.org/10.1175/ 1520-0493(1980)108<1626:TICATP > 2.0.CO;2.

Brown, R. A., and V. T. Wood, 2012: The tornadic vortex signature: An update. Wea. Forecasting, 27, 525-530, https://doi.org/ 10.1175/WAF-D-11-00111.1.

— L L. R. Lemon, and D. W. Burgess, 1978: Tornado detection by pulsed Doppler radar. Mon. Wea. Rev., 106, 29-38, https://doi.org/ 10.1175/1520-0493(1978)106<0029:TDBPDR >2.0.CO;2.

Browning, K. A., and F. H. Ludlam, 1962: Airflow in convective storms. Quart. J. Roy. Meteor. Soc., 88, 117-135, https:// doi.org/10.1002/qj.49708837602.

Bunkers, M. J., and M. A. Baxter, 2011: Radar tornadic debris signatures on 27 April 2011. Electron. J. Oper. Meteor., 12 (7), http://nwafiles.nwas.org/ej/pdf/2011-ION1.pdf.

Burgess, D. W., and R. A. Brown, 1973: The structure of a severe right-moving thunderstorm: New single Doppler radar evidence. Preprints, Eighth Conf. on Severe Local Storms, Boston, MA, Amer. Meteor. Soc., 40-43.

, and M. A. Magsig, 1998: Recent observations of tornado development at near range to WSR-88D radars. Preprints, 19th Conf. on Severe Local Storms, Minneapolis, MN, Amer. Meteor. Soc., 756-759.

- _ _ J. Wurman, D. C. Dowell, and Y. Richardson, 2002: Radar observations of the 3 May 1999 Oklahoma City tornado. Wea. Forecasting, 17, 456-471, https://doi.org/10.1175/ 1520-0434(2002)017<0456:ROOTMO > 2.0.CO;2.

— E. R. Mansell, C. M. Schwarz, and B. J. Allen, 2010: Tornado and tornadogenesis events seen by the NOXP X-band, dualpolarization radar during VORTEX2 2010. Preprints, 25th Conf. on Severe Local Storms, Denver, CO, Amer. Meteor. Soc., 5.2, http://ams.confex.com/ams/pdfpapers/176164.pdf. 
, B. R. Smith, H. B. Bluestein, Z. B. Wienhoff, D. W. Reif, R. Smith, and D. Speheger, 2016: A mini-supercell observed during an outbreak of classic supercells on 9 May 2013. 28th Conf. on Severe Local Storms, Portland, OR, Amer. Meteor. Soc., 12A.1, https://ams.confex.com/ams/28SLS/webprogram/ Paper300945.html.

Cho, J. Y. N., and J. M. Kurdzo, 2019: Tornado-related benefit modeling for weather radar networks. 35th Conf. on Environmental Information Processing Technologies, Phoenix, AZ, Amer. Meteor. Soc. 10B.1, https://ams.confex.com/ams/2019Annual/ meetingapp.cgi/Paper/348867.

Chrisman, J., 2014a: Multiple Elevation Scan Option for SAILS (MESO-SAILS): The next step in dynamic scanning for the WSR-88D. NOAA, 27 pp., https://www.roc.noaa.gov/WSR88D/ PublicDocs/NewTechnology/MESO-SAILS_Description_Briefing Jan_2014.pdf.

- 2014b: The continuing evolution of dynamic scanning. NEXRAD Now, No. 23, NOAA/NWS/Radar Operations Center, Norman, OK, 8-13, http://www.roc.noaa.gov/WSR88D/ PublicDocs/NNOW/NNow23a.pdf.

Christian, T. W., and R. M. Wakimoto, 1989: The relationship between radar reflectivities and clouds associated with horizontal roll convection on 8 August 1982. Mon. Wea. Rev., 117, 1530-1544, https://doi.org/10.1175/1520-0493(1989)117<1530: TRBRRA $>2.0 . \mathrm{CO} ; 2$.

Donaldson, R. J., 1970: Vortex signature recognition by a Doppler radar. J. Appl. Meteor., 9, 661-670, https://doi.org/10.1175/ 1520-0450(1970)009<0661:VSRBAD > 2.0.CO;2.

Droegemeier, K., and R. Wilhelmson, 1986: Kelvin-Helmholtz instability in a numerically simulated thunderstorm outflow. Bull. Amer. Meteor. Soc., 67 (4), 416-417.

French, M. M., H. B. Bluestein, I. PopStefanija, C. A. Baldi, and R. T. Bluth, 2013: Reexamining the vertical development of tornadic vortex signatures in supercells. Mon. Wea. Rev., 141, 4576-4601, https://doi.org/10.1175/MWR-D-12-00315.1.

Fujita, T., 1958: Mesoanalysis of the Illinois tornadoes of 9 April 1953. J. Meteor., 15, 288-296, https://doi.org/10.1175/15200469(1958)015<0288:MOTITO > 2.0.CO;2.

Griffin, C. B., D. J. Bodine, and R. D. Palmer, 2017: Kinematic and polarimetric radar observations of the 10 May 2010, Moore-Choctaw, Oklahoma, tornadic debris signature. Mon. Wea. Rev., 145, 2723-2741, https://doi.org/10.1175/ MWR-D-16-0344.1.

_ C. C. Weiss, A. E. Reinhart, J. C. Snyder, H. B. Bluestein, J. Wurman, and K. A. Kosiba, 2018: In situ and radar observations of the low reflectivity ribbon in supercells during VORTEX2. Mon. Wea. Rev., 146, 307-327, https://doi.org/ 10.1175/MWR-D-17-0201.1.

Heinselman, P. L., and S. M. Torres, 2011: High-temporal-resolution capabilities of the National Weather Radar Testbed Phased-Array Radar. J. Appl. Meteor. Climatol., 50, 579-593, https://doi.org/10.1175/2010JAMC2588.1.

- D. L. Priegnitz, K. L. Manross, T. M. Smith, and R. W. Adams, 2008: Rapid sampling of severe storms by the National Weather Radar Testbed Phased Array Radar. Wea. Forecasting, 23, 808824, https://doi.org/10.1175/2008WAF2007071.1.

— - D. LaDue, D. M. Kingfield, and R. Hoffman, 2015: Tornado warning decisions using phased-array radar data. Wea. Forecasting, 30, 57-78, https://doi.org/10.1175/WAF-D-1400042.1.

Houser, J. L., H. B. Bluestein, and J. C. Snyder, 2016: A finescale radar examination of the tornadic debris signature and weakecho reflectivity band associated with a large, violent tornado.
Mon. Wea. Rev., 144, 4101-4130, https://doi.org/10.1175/ MWR-D-15-0408.1.

Ice, R. L., and Coauthors, 2011: Sensitivity of operational weather radars. 27th Conf. on Interactive Information Processing Systems, Seattle, WA, Amer. Meteor. Soc., 370, https://ams.confex.com/ ams/91Annual/webprogram/Paper183654.html.

Isom, B., and Coauthors, 2013: The Atmospheric Imaging Radar: Simultaneous volumetric observations using a phased array weather radar. J. Atmos. Oceanic Technol., 30, 655-675, https://doi.org/10.1175/JTECH-D-12-00063.1.

Jedlovec, G. J., U. Nair, and S. L. Haines, 2006: Detection of storm damage tracks with EOS data. Wea. Forecasting, 21, 249-267, https://doi.org/10.1175/WAF923.1.

Kessinger, C. J., D. B. Parsons, and J. W. Wilson, 1988: Observations of a storm containing misocyclones, downbursts, and horizontal vortex circulations. Mon. Wea. Rev., 116, 1959-1982, https:// doi.org/10.1175/1520-0493(1988)116<1959:OOASCM >2.0.CO;2.

Kingfield, D. M., and K. M. de Beurs, 2017: Landsat identification of tornado damage by land cover and an evaluation of damage recovery in forests. J. Appl. Meteor. Climatol., 56, 965-987, https://doi.org/10.1175/JAMC-D-16-0228.1.

Kosiba, K., J. Wurman, Y. Richardson, P. Markowski, P. Robinson, and J. Marquis, 2013: Genesis of the Goshen County, Wyoming, tornado on 5 June 2009 during VORTEX2. Mon. Wea. Rev., 141, 1157-1181, https://doi.org/10.1175/MWR-D-12-00056.1.

Kumjian, M. R., A. V. Ryzhkov, V. M. Melnikov, and T. J. Schuur, 2010: Rapid-scan super-resolution observations of a cyclic supercell with a dual-polarization WSR-88D. Mon. Wea. Rev., 138, 3762-3786, https://doi.org/10.1175/2010MWR3322.1.

Kurdzo, J. M., D. J. Bodine, B. L. Cheong, and R. D. Palmer, 2015: High-temporal resolution polarimetric X-band Doppler radar observations of the 20 May 2013 Moore, Oklahoma, tornado. Mon. Wea. Rev., 143, 2711-2735, https://doi.org/10.1175/MWRD-14-00357.1.

— , and Coauthors, 2017: Observations of severe local storms and tornadoes with the Atmospheric Imaging Radar. Bull. Amer. Meteor. Soc., 98, 915-935, https://doi.org/10.1175/BAMS-D15-00266.1.

Kuster, C. M., P. L. Heinselman, and M. Austin, 2015: 31 May 2013 El Reno tornadoes: Advantages of rapid-scan phased-array radar data from a warning forecaster's perspective. Wea. Forecasting, 30, 933-956, https://doi.org/10.1175/WAF-D-14-00142.1.

$\longrightarrow,-$, and T. J. Schuur, 2016: Rapid-update radar observations of downbursts occurring within an intense multicell thunderstorm on 14 June 2011. Wea. Forecasting, 31, 827-851, https://doi.org/10.1175/WAF-D-15-0081.1.

— J. C. Snyder, T. J. Schuur, T. T. Lindley, P. L. Heinselman, J. C. Furtado, J. W. Brogden, and R. Toomey, 2019: Rapidupdate radar observations of $\mathrm{Z}_{\mathrm{DR}}$ column depth and its use in the warning decision process. Wea. Forecasting, 34, 1173-1188, https://doi.org/10.1175/WAF-D-19-0024.1.

Lakshmanan, V., T. Smith, G. J. Stumpf, and K. Hondl, 2007: The Warning Decision Support System-Integrated Information. Wea. Forecasting, 22, 592-608, https://doi.org/10.1175/WAF1009.1.

Lemon, L. R., and C. A. Doswell, 1979: Severe thunderstorm evolution and mesocyclone structure as related to tornadogenesis. Mon. Wea. Rev., 107, 1184-1197, https://doi.org/ 10.1175/1520-0493(1979)107<1184:STEAMS>2.0.CO;2.

—, R. J. Donaldson, D. W. Burgess, and R. A. Brown, 1977: Doppler radar application to severe thunderstorm study and potential real-time warning. Bull. Amer. Meteor. Soc., 58, 1187-1193, https://doi.org/10.1175/1520-0477(1977)058<1187: DRATST $>2.0 . \mathrm{CO} ; 2$. 
Lhermitte, R. M., 1964: Doppler radars as severe storm sensors. Bull. Amer. Meteor. Soc., 45, 587-596, https://doi.org/10.1175/ 1520-0477-45.9.587.

Markowski, P. M., 2002: Hook echoes and rear-flank downdrafts: A review. Mon. Wea. Rev., 130, 852-876, https://doi.org/10.1175/ 1520-0493(2002)130<0852:HEARFD>2.0.CO;2.

McLaughlin, D., and Coauthors, 2009: Short-wavelength technology and the potential for distributed networks of small radar systems. Bull. Amer. Meteor. Soc., 90, 1797-1818, https:// doi.org/10.1175/2009BAMS2507.1.

Molthan, A. L., J. R. Bell, T. A. Cole, and J. E. Burks, 2014: Satellite-based identification of tornado damage tracks from the 27 April 2011 severe weather outbreak. J. Oper. Meteor., 2, 191-208, https://doi.org/10.15191/nwajom.2014.0216.

NOAA Comprehensive Large Array-Data Stewardship System (CLASS), 2016: GOES Satellite Data-Imager (GVAR_IMG) Archive. NOAA, accessed 4 February 2017, https://www. avl.class.noaa.gov/saa/products/search?datatype_family $=$ GVAR_IMG.

NOAA/NWS Radar Operations Center, 1991: NOAA Next Generation Radar (NEXRAD) Level 2 Base Data. NOAA/Nationa Centers for Environmental Information, subset used: 9-10 May 2016, accessed 9 September 2016, https://doi.org/10.7289/ V5W9574V.

OFCM, 2016: WSR-88D meteorological observations. Part A: System concepts, responsibilities, and procedures. Federal Meteorological Handbook 11, U.S. Department of Commerce/NOAA, FCMH11A-2016, 25 pp., https://www.ofcm.gov/publications/fmh/ FMH11/2016FMH11PTA.pdf.

Oliveira, M. I., M. Xue, B. J. Roberts, L. J. Wicker, and N. Yussouf, 2019: Horizontal vortex tubes near a simulated tornado: Three-dimensional structure and kinematics. Atmosphere, $\mathbf{1 0}$, 716, https://doi.org/10.3390/atmos10110716.

Orf, L., R. Wilhelmson, B. Lee, C. Finley, and A. Houston, 2017: Evolution of a long-track violent tornado within a simulated supercell. Bull. Amer. Meteor. Soc., 98, 45-68, https://doi.org/ 10.1175/BAMS-D-15-00073.1.

Pazmany, A. L., J. B. Mead, H. B. Bluestein, J. C. Snyder, and J. B. Houser, 2013: A mobile rapid-scanning X-band polarimetric (RaXPol) Doppler radar system. J. Atmos. Oceanic Technol., 30, 1398-1413, https://doi.org/10.1175/JTECH-D 12-00166.1.

Reinhart, A. E., and D. M. Kingfield, 2018: The role of WSR-88D VCPs and intra-volume scanning strategies on volumetric analyses of thunderstorm intensity using simulation observations. 29th Conf. on Weather Analysis and Forecasting, Denver, CO, Amer. Meteor. Soc., 11A.1, https://ams.confex.com/ams/ 29WAF25NWP/webprogram/Paper345509.html

Ryzhkov, A. V., T. J. Schuur, D. W. Burgess, and D. S. Zrnić, 2005: Polarimetric tornado detection. J. Appl. Meteor., 44, 557-570, https://doi.org/10.1175/JAM2235.1.

Scharfenberg, K. A., and Coauthors, 2005: The Joint Polarization Experiment: Polarimetric radar in forecasting and warning decision making. Wea. Forecasting, 20, 775-788, https://doi.org/ 10.1175/WAF881.1.

Schultz, C. J., and Coauthors, 2012: Dual-polarization tornadic debris signatures. Part I: Examples and utility in an operational setting. Electron. J. Oper. Meteor., 13, 120-137, http:// nwafiles.nwas.org/ej/pdf/2012-EJ9.pdf.

Scofield, R. A., and J. F. W. Purdom, 1986: The use of satellite data for mesoscale analyses and forecasting applications Mesoscale Meteorology and Forecasting, P. S. Ray, Ed., Amer. Meteor. Soc., 118-150.
Snyder, J. C., and H. B. Bluestein, 2014: Some considerations for the use of high-resolution mobile radar data in tornado intensity determination. Wea. Forecasting, 29, 799-827, https:// doi.org/10.1175/WAF-D-14-00026.1.

, and A. V. Ryzhkov, 2015: Automated detection of polarimetric tornadic debris signatures using a hydrometeor classification algorithm. J. Appl. Meteor. Climatol., 54, 1861-1870, https://doi.org/10.1175/JAMC-D-15-0138.1.

$\longrightarrow$, H. B. Bluestein, G. Zhang, and S. J. Frasier, 2010: Attenuation correction and hydrometeor classification of high-resolution, $\mathrm{X}$-band, dual-polarized mobile radar measurements in severe convective storms. J. Atmos. Oceanic Technol., 27, 1979-2001, https://doi.org/10.1175/2010JTECHA1356.1.

,,-- V. Venkatesh, and S. Frasier, 2013: Observations of polarimetric signatures in supercells by an X-band mobile Doppler radar. Mon. Wea. Rev., 141, 3-29, https://doi.org/ 10.1175/MWR-D-12-00068.1.

A. V. Ryzhkov, M. R. Kumjian, A. P. Khain, and J. Picca, 2015: A $Z_{\mathrm{DR}}$ column detection algorithm to examine convective storm updrafts. Wea. Forecasting, 30, 1819-1844, https://doi.org/10.1175/WAF-D-15-0068.1.

Tanamachi, R. L., and P. L. Heinselman, 2016: Rapid-scan, polarimetric observations of central Oklahoma severe storms on 31 May 2013. Wea. Forecasting, 31, 19-42, https://doi.org/ 10.1175/WAF-D-15-0111.1.

A. T. LaFleur, M. Sharma, S. J. Frasier, W. Heberling, and C. Wolsieffer, 2018: Observations of severe storms by a novel, polarimetric, phased array mobile radar. 29th Conf. on Severe Local Storms, Stowe, VT, Amer. Meteor. Soc., 15.4, https:// ams.confex.com/ams/29SLS/webprogram/Paper348420.html.

Thompson, R. L., and R. Edwards, 2000: An overview of environmental conditions and forecast implications of the 3 May 1999 tornado outbreak. Wea. Forecasting, 15, 682-699, https:// doi.org/10.1175/1520-0434(2000)015<0682:AOOECA > 2.0. $\mathrm{CO} ; 2$

,-- , J. A. Hart, K. L. Elmore, and P. Markowski, 2003: Close proximity soundings within supercell environments obtained from the Rapid Update Cycle. Wea. Forecasting, 18, 1243-1261, https:// doi.org/10.1175/1520-0434(2003)018<1243:CPSWSE >2.0.CO;2.

Trapp, R. J., 1999: Observations of nontornadic low-level mesocyclones and attendant tornadogenesis failure during VORTEX. Mon. Wea. Rev., 127, 1693-1705, https://doi.org/10.1175/15200493(1999)127<1693:OONLLM>2.0.CO;2.

Van Den Broeke, M. S., 2015: Polarimetric tornadic debris signature variability and debris fallout signatures. J. Appl. Meteor. Climatol., 54, 2389-2405, https://doi.org/10.1175/ JAMC-D-15-0077.1.

- and S. T. Jauernic, 2014: Spatial and temporal characteristics of polarimetric tornadic debris signatures. J. Appl. Meteor. Climatol., 53, 2217-2231, https://doi.org/10.1175/JAMC-D-140094.1.

Wade, A. R., M. C. Coniglio, and C. L. Ziegler, 2018: Comparison of near- and far-field supercell inflow environments using radiosonde observations. Mon. Wea. Rev., 146, 2403-2415, https://doi.org/10.1175/MWR-D-17-0276.1.

Wakimoto, R. M., N. T. Atkins, K. M. Butler, H. B. Bluestein, K. Thiem, J. Snyder, and J. Houser, 2015: Photogrammetric analysis of the $2013 \mathrm{El}$ Reno tornado combined with mobile X-band polarimetric radar data. Mon. Wea. Rev., 143, 26572683, https://doi.org/10.1175/MWR-D-15-0034.1.

- and Coauthors, 2016: Aerial damage survey of the $2013 \mathrm{El}$ Reno tornado combined with mobile radar data. Mon. Wea. Rev., 144, 1749-1776, https://doi.org/10.1175/MWR-D-15-0367.1. 
Weber, M. E., J. Y. Cho, J. S. Herd, J. M. Flavin, W. E. Benner, and G. S. Torok, 2007: The next-generation multimission U.S. surveillance radar network. Bull. Amer. Meteor. Soc., 88, 1739-1752, https://doi.org/10.1175/BAMS-88-11-1739.

Weckwerth, T. M., J. W. Wilson, R. M. Wakimoto, and N. A. Crook, 1997: Horizontal convective rolls: Determining the environmental conditions supporting their existence and characteristics. Mon. Wea. Rev., 125, 505-526, https://doi.org/10.1175/15200493(1997)125<0505:HCRDTE > 2.0.CO;2.

- T. W. Horst, and J. W. Wilson, 1999: An observational study of the evolution of horizontal convective rolls. Mon. Wea. Rev., 127, 2160-2179, https://doi.org/10.1175/1520-0493(1999) 127<2160:AOSOTE $>2.0$. CO;2.

Weiss, C. C., J. L. Schroeder, J. Guynes, P. S. Skinner, and J. Beck, 2009: The TTUKa mobile Doppler radar: Coordinated radar and in situ measurements of supercell thunderstorms during Project VORTEX2.34th Conf. on Radar Meteor., Williamsburg, VA, Amer. Meteor. Soc. 11B.2, http://ams.confex.com/ams/ 34Radar/techprogram/paper_155425.htm.

Wilson, K. A., P. L. Heinselman, C. M. Kuster, D. M. Kingfield, and Z. Kang, 2017a: Forecaster performance and workload: Does radar update time matter? Wea. Forecasting, 32, 253-274, https://doi.org/10.1175/WAF-D-16-0157.1.

,$- \longrightarrow$, and — 2017b: Considerations for phased-array radar data use within the National Weather Service. Wea. Forecasting, 32, 1959-1965, https://doi.org/10.1175/WAF-D-17-0084.1.

Witt, A., D. W. Burgess, A. Seimon, J. T. Allen, J. C. Snyder, and H. B. Bluestein, 2018: Rapid-scan radar observations of an Oklahoma tornadic hailstorm producing giant hail. Wea. Forecasting, 33, 1263-1282, https://doi.org/10.1175/WAF-D18-0003.1.
Wolfinbarger, M., J. R. Greenfield, T. A. Cannon, and W. G. McPherson Jr., 2004: WeatherScope: A streamlined display application for geographic weather data. Preprints, 20th Int. Conf. on Interactive Information and Processing Systems (IIPS) for Meteorology, Oceanography, and Hydrology, Boulder, CO, Amer. Meteor. Soc., 13.3, https://ams.confex.com/ams/84Annual/ techprogram/paper_73640.htm.

Wood, V. T., and R. A. Brown, 1997: Effects of radar sampling on single-Doppler velocity signatures of mesocyclones and tornadoes. Wea. Forecasting, 12, 928-938, https://doi.org/10.1175/ 1520-0434(1997)012<0928:EORSOS > 2.0.CO;2.

WSEC, 2006: A recommendation for an enhanced Fujita scale (EFscale). Texas Tech University Wind Science and Engineering Center Rep., 108 pp.

Wurman, J., and K. Kosiba, 2013: Finescale radar observations of tornado and mesocyclone structures. Wea. Forecasting, 28 , 1157-1174, https://doi.org/10.1175/WAF-D-12-00127.1.

_ J. Straka, E. Rasmussen, M. Randall, and A. Zahrai, 1997: Design and deployment of a portable, pencil-beam, pulsed, 3-cm Doppler radar. J. Atmos. Oceanic Technol., 14, 1502-1512, https://doi.org/ 10.1175/1520-0426(1997)014<1502:DADOAP > 2.0.CO;2.

—, D. Dowell, Y. Richardson, P. Markowski, E. Rasmussen, D. Burgess, L. Wicker, and H. Bluestein, 2012: The second Verification of the Origins of Rotation in Tornadoes Experiment: VORTEX2. Bull. Amer. Meteor. Soc., 93, 1147-1170, https:// doi.org/10.1175/BAMS-D-11-00010.1.

Yuan, M., M. Dickens-Micozzi, and M. A. Magsig, 2002: Analysis of tornado damage tracks from the 3 May tornado outbreak using multispectral satellite imagery. Wea. Forecasting, 17, 382-398, https://doi.org/10.1175/1520-0434(2002)017<0382: AOTDTF $>2.0 . \mathrm{CO} ; 2$. 